\title{
Facilitated Nucleocytoplasmic Shuttling of the Ran Binding Protein RanBP1
}

\author{
KENDRA PLAFKER AND IAN G. MACARA* \\ Markey Center for Cell Signaling and Department of Pharmacology, \\ University of Virginia, Charlottesville, Virginia 22908
}

Received 7 September 1999/Returned for modification 12 October 1999/Accepted 21 February 2000

\begin{abstract}
The Ran binding protein RanBP1 is localized to the cytosol of interphase cells. A leucine-rich nuclear export signal (NES) near the $C$ terminus of RanBP1 is essential to maintain this distribution. We now show that RanBP1 accumulates in nuclei of cells treated with the export inhibitor, leptomycin B, and collapse of the nucleocytoplasmic Ran:GTP gradient leads to equilibration of RanBP1 across the nuclear envelope. Low temperature prevents nuclear accumulation of RanBP1, suggesting that import does not occur via simple diffusion. Glutathione $S$-transferase (GST)-RanBP1(1-161), which lacks the NES, accumulates in the nucleus after cytoplasmic microinjection. In permeabilized cells, nuclear accumulation of GST-RanBP1(1-161) requires nuclear Ran:GTP but is not inhibited by a dominant interfering G19V mutant of Ran. Nuclear accumulation is enhanced by addition of exogenous karyopherins/importins or RCC1, both of which also enhance nuclear Ran accumulation. Import correlates with Ran concentration. Remarkably, an E37K mutant of RanBP1 does not import into the nuclei under any conditions tested despite the fact that it can form a ternary complex with Ran and importin $\beta$. These data indicate that RanBP1 translocates through the pores by an active, nonclassical mechanism and requires Ran:GTP for nuclear accumulation. Shuttling of RanBP1 may function to clear nuclear pores of Ran:GTP, to prevent premature release of import cargo from transport receptors.
\end{abstract}

The defining feature of eukaryotic cells is the compartmentalization of DNA replication and transcription within the nucleus. Access to the nuclear compartment is provided by pores that are plugged through the double membrane of the nuclear envelope (for reviews, see references 15 and 66). A multitude of soluble transport receptors and accessory proteins controls the transit of protein and nucleic acid cargo through these pores (for recent reviews, see references 24, 40, 42, and 64). The vectoriality of cargo transport is in many cases dependent on the asymmetric distribution of Ran, a small GTP binding protein, and of its regulatory factors $(8,17)$. Ran is predominantly nuclear and is believed to be maintained in the GTPbound state (Ran:GTP) by a guanine nucleotide exchange factor, RCC1, which is associated with chromatin in the nucleus $(7,58)$. A Ran-specific GTPase-activating protein, RanGAP, which converts Ran to the GDP-bound state, is excluded from the nucleus $(5,13,30)$. This asymmetric arrangement of regulatory factors ensures that a steep Ran:GTP gradient exists across the nuclear pores, and the collapse of this gradient inhibits many forms of nuclear traffic $(32,52)$.

Proteins destined for import into the nucleus frequently possess a nuclear localization signal (NLS) containing multiple basic amino acid residues (16). The NLS is recognized by an adapter protein called importin $\alpha$ (also variously called karyopherin- $\alpha$, p56, SRP1, PTAC58, and pendulin) which associates with a transporter module, importin $\beta$ (also called karyopherin- $\beta$ and p97) $(11,25,31,36,48,61)$. This complex can bind to nuclear pores and translocate from one side of the pore to the other, probably by a type of facilitated diffusion $(18,50)$. Ran:GTP, present within the nucleus, dissociates the complex from the pores and releases importin $\alpha$ and its associated cargo from importin $\beta(26,49)$. Thus, it may be that the Ran gradient

* Corresponding author. Mailing address: Room 7191 Hospital West, P.O. Box 800577, HSC, University of Virginia School of Medicine, Charlottesville, VA 22908. Phone: (804) 982-0074. Fax: (804) 924-1236. E-mail: imacara@virginia.edu. is not required for translocation per se but can drive a net accumulation of the cargo in the nuclear compartment.

The importin $\beta$ is then believed to return to the cytosol as a complex with Ran:GTP $(26,28)$. There, the RanGAP must hydrolyze the GTP to permit release of the importin $\beta$ for another round of import. However, RanGAP alone is unable to act on Ran:GTP that is bound to importin $\beta(19,26)$. Another cytosolic factor, the Ran binding protein RanBP1, is required together with importin $\alpha$ to permit efficient GTP hydrolysis by RanGAP $(4,20)$. The giant nucleoporin Nup358 can probably serve a similar function $(65,67)$. RanBP1 is a 23-kDa cytosolic protein that possesses a conserved Ran binding domain (RanBD) of about 135 amino acid residues, which specifically recognizes Ran in the GTP-bound state $(3,14)$. The crystal structure of Ran in a complex with the second RanBD of Nup358 has been solved, and the two proteins form an unusually tight embrace in which the $\mathrm{N}$ terminus of the RanBD is wrapped around Ran, and the C terminus of Ran is wrapped around the RanBD (62). Ran:GTP can form a ternary complex with RanBP1 and importin $\beta$ or with other members of the karyopherin family, and this complex is only weakly susceptible to RanGAP $(4,38)$. The presence of importin $\alpha$, which will sequester free importin $\beta$, increases the efficiency of the hydrolysis $(4,20)$. RanBP1 is therefore predicted to be required for continued nuclear protein import, and this has been shown to be the case in yeast (57). RanBP1 and importin $\beta$ have no measurable affinity for each other or-separatelyfor Ran:GDP, but surprisingly they can form a stable ternary complex with one another (12). The purpose of this RanBP1importin $\beta$-Ran:GDP complex remains obscure.

Proteins that need to be exported from the nucleus often possess a distinct, leucine-rich nuclear export signal (NES) (for a review, see reference 23). The NES can form a ternary complex with an export receptor, called Crm1 (or exportin 1), and Ran:GTP $(2,21,22,44,60)$. The formation of this complex will therefore be favored in the nucleus. After translocation through the pores, RanGAP — again with the likely assistance 
of RanBP1-can hydrolyze the Ran:GTP and release the NES cargo into the cytosol. Therefore, RanBP1 probably plays an essential role in nuclear protein export. A similar mechanism can account for the export of importin $\alpha$ by the transport factor Cas (37).

A complete understanding of nuclear protein transport requires that we determine the mechanism by which essential factors such as RanBP1 maintain their specific locations within the cell. The role of RanBP1 as an accessory factor for RanGAP implies that RanBP1 must be retained in the cytosol. Indeed, ectopic expression of nuclear RanBP1 is toxic to cells, and microinjection of RanBP1 into nuclei inhibits nuclear transport, probably because it sequesters nuclear Ran:GTP and effectively collapses the nucleocytoplasmic Ran:GTP gradient $(32,53)$. It may also interfere with the formation of export complexes. This requirement for a cytoplasmic location presents a problem to the cell, because RanBP1 is in principle small enough to diffuse through the nuclear pores, and within the nucleus it will form a stable ternary complex with Ran:GTP and importin $\beta$. Two distinct mechanisms may confer the cytoplasmic distribution of small proteins: (i) retention by binding to a larger cytoplasmic protein and (ii) active export. RanBP1 possesses a functional NES near its $\mathrm{C}$ terminus $(54,68)$. However, it also possesses an adjacent upstream sequence that may act in cytoplasmic retention (54). Three other groups have provided indications that RanBP1 may shuttle between the cytosol and nucleus. Zolotukhin and Felber (68) found that the ectopic expression of the Rev protein (which contains an NES) can lead to the nuclear accumulation of RanBP1, presumably by competing for Crm1. Schlenstedt et al. (56), in a study of the Yrb4p importin in yeast, demonstrated that a dominant interfering mutant of Yrb4p that blocks bidirectional nuclear transport also caused the nuclear accumulation of Yrb1p, the yeast homologue of RanBP1. However, in both cases the inhibitory protein was expressed for a period greater than the cell cycle time. Thus, these results do not resolve whether RanBP1 continually shuttles across the nuclear pores or instead enters the nucleus only at a specific time during cell division. For example, in mammalian cells an NES might be required to ensure that RanBP1 is excluded from the nucleus after mitosis when the integrity of the nuclear envelope is reestablished. A third study examined the distribution of RanBP1 in Xenopus oocytes after microinjection of an NES-peptide conjugate, which inhibits mRNA and snRNA transport (46). Oocytes were fractionated after $6.5 \mathrm{~h}$, and the nuclear and cytosolic fractions were immunoblotted for RanBP1. These results demonstrated that RanBP1 became partially associated with the nuclei-either at the pores or within the nucleoplasm-in the presence of the competing NES-peptide. In all of these studies, however, it could be argued that the RanBP1 enters the nucleus only in response to a general perturbation in nuclear transport.

We now show that RanBP1 does continually shuttle quite rapidly between the nuclear and cytoplasmic compartments. Leptomycin B (LMB), which specifically inhibits NES-mediated export (63), causes a nuclear accumulation of endogenous RanBP1 and of a green fluorescent protein (GFP)-RanBP1 fusion protein. Microinjected GST-RanBP1 lacking its C-terminal NES accumulates in the nucleus in the absence of any transport inhibitors. Collapse of the Ran:GTP gradient results in the equilibration of RanBP1 across the nuclear envelope. The nuclear accumulation of a GST-RanBP1 mutant that lacks the NES requires nuclear Ran:GTP. A point mutation in the $\mathrm{N}$-terminal arm of the RanBD of RanBP1 completely blocks import into nuclei under the conditions tested. Although this mutant has a reduced affinity for Ran, it can form a ternary complex with Ran:GTP and importin $\beta$ or importin 5 (47). We propose that the RanBP1 can translocate through the nuclear pores by an active mechanism and, in the absence of functional export, accumulate within the nuclei in a complex with Ran: GTP.

\section{MATERIALS AND METHODS}

Proteins. Recombinant proteins used in in vitro import assays and microinjection assays were overexpressed in Escherichia coli as glutathione $S$-transferase (GST) fusions and affinity purified using glutathione (GSH)-Sepharose (Pharmacia catalog no. 17-0756-01). The proteins were either eluted off the Sepharose with GSH or proteolytically cleaved away from the GST using thrombin, as described previously $(38,39)$. RanBP1 mutants were isolated from mutant libraries generated either by template-limited PCR or by incorporation of doped oligonucleotides (47). Crm1 was partially purified as described by Holaska and Paschal (29). Recombinant Ran was converted to the GTP-bound state (Ran: GTP) by incubation with $1 \mathrm{mM}$ GTP in the presence of recombinant RCC1 (2.4 $\mu \mathrm{g} / \mathrm{ml})$ and $5 \mathrm{mM} \mathrm{MgCl}_{2}\left(10 \mathrm{~min}\right.$ at $\left.30^{\circ} \mathrm{C}\right)$ or by incubation with $1 \mathrm{mM}$ GTP in $25 \mathrm{mM}$ morpholinepropanesulfonic acid (MOPS; $\mathrm{pH}$ 7.1)-1 mM EDTA for 30 min on ice before the addition of $1 \mathrm{mM} \mathrm{MgCl}_{2}$. Proteins were exchanged into import assay buffer or microinjection buffer, as needed, by size exclusion chromatography using Pharmacia PD10 columns.

Plasmids. Various recombinant DNAs were used in transfections and transformations, some of which have been described previously $(10,41,53)$. The following constructs were made specifically for this study. Vectors encoding a fusion of GFP to the $\mathrm{N}$ termini of various RanBP1 mutants (E37K, G71C/K76E, $\mathrm{R} 91 \mathrm{~S} / \mathrm{K} 97 \mathrm{~T}$, and R92K/D93Y) were constructed by digesting the appropriate pGEX-RanBP1 plasmids with Bam $\mathrm{HI}$ and EcoRI and ligating the insert into the same restriction sites of the pKGFP vector (10). pKGFP-GFP-RanBP1 was constructed by inserting a BamHI/EcoRI fragment encoding GFP-RanBP1 into pKGFP. A pGEX-importin- $\beta(45-462)$ vector was constructed by amplification of the required fragment of importin $\beta$ by the PCR, using $5^{\prime}$ and $3^{\prime}$ primers possessing Bam HI and SacI sites, respectively. The insert was moved into pGEX-KG to produce a plasmid that encodes GST-importin $\beta(45-462)$.

Microinjections. Baby hamster kidney (BHK-21) cells were plated onto Cellocate gridded coverslips (Eppendorf catalog no. 930002046); $48 \mathrm{~h}$ later, the growth medium was removed and replaced with Ringer's solution $(25 \mathrm{mM}$ HEPES [pH 7.2], $110 \mathrm{mM} \mathrm{NaCl}, 5 \mathrm{mM} \mathrm{KCl}, 2 \mathrm{mM} \mathrm{CaCl}_{2}, 1 \mathrm{mM} \mathrm{MgSO}_{4}, 10 \mathrm{mM}$ glucose, $1 \mathrm{mM} \mathrm{KH}{ }_{2} \mathrm{PO}_{4}, 1 \mathrm{mg}$ of bovine serum albumin (BSA)/ml), and the nuclei of the cells were injected with the indicated recombinant proteins and fluorescein isothiocyanate (FITC)-coupled dextran in microinjection buffer (10 $\mathrm{mM} \mathrm{NaPO}_{4}, 70 \mathrm{mM} \mathrm{KCl}, 1 \mathrm{mM} \mathrm{MgCl}$ [pH 7.2]). Cells were then incubated at $37^{\circ} \mathrm{C}$ for 10 to $30 \mathrm{~min}$, fixed, permeabilized, and subjected to immunofluorescence as described.

Transfections. Plasmid DNAs were transfected into BHK-21 cells by the calcium phosphate method. Cells were grown at $37^{\circ} \mathrm{C}$ in Dulbecco modified Eagle medium containing $10 \%$ heat-inactivated calf serum and penicillin-streptomycin; $24 \mathrm{~h}$ posttransfection, the medium was replaced with fresh medium and incubated at $37^{\circ} \mathrm{C}$ for another 24 to $48 \mathrm{~h}$. Transfected cells were either untreated, placed on ice, or incubated in the presence of $200 \mathrm{nM}$ LMB. After the appropriate treatments, the cells were fixed and permeabilized with $4 \%$ paraformaldehyde in phosphate-buffered saline (PBS; $137 \mathrm{mM} \mathrm{NaCl}, 3 \mathrm{mM} \mathrm{KCl}, 10 \mathrm{mM} \mathrm{Na}{ }_{2} \mathrm{HPO}_{4}, 2$ $\mathrm{mM} \mathrm{KH} \mathrm{PO}_{4}$ [pH 7.4]) and ice-cold methanol as described previously (10). Fixed cells were then prepared for viewing by fluorescence microscopy.

In vitro import assays. Assays using permeabilized cells were performed following established protocols (1), with some modification. BHK-21 cells were plated onto glass coverslips coated with poly-L-lysine. At 20 to $24 \mathrm{~h}$ after plating, cells were permeabilized with $0.008 \%$ digitonin in assay buffer $(\mathrm{AB} ; 20 \mathrm{mM}$ HEPES-KOH [pH 7.6], potassium acetate, $80 \mathrm{mM} 4 \mathrm{mM}$ magnesium acetate, $250 \mathrm{mM}$ sucrose, $1 \mathrm{mM}$ dithiothreitol) for $5 \mathrm{~min}$ on ice. After permeabilization, cells were overlaid with an energy-regenerating system $(10 \mathrm{mM}$ creatine phosphate, $50 \mathrm{mg}$ of creatine kinase $/ \mathrm{ml}, 500 \mu \mathrm{M}$ ATP, $500 \mu \mathrm{M}$ GTP) and rabbit reticulocyte lysate (Promega catalog no. L4151) or HeLa cell lysate and/or purified recombinant factors, incubated at room temperature for 5 to $30 \mathrm{~min}$, washed with $\mathrm{AB}$, fixed with $4 \%$ paraformaldehyde in PBS at room temperature for $15 \mathrm{~min}$, permeabilized with $-20^{\circ} \mathrm{C}$ methanol for $2 \mathrm{~min}$, and prepared for fluorescence microscopy as described below.

Fluorescence microscopy. After fixation and permeabilization, cells which contained GFP fusions were incubated with $10 \mathrm{ng}$ of 4',6-diamidino-2-phenylindole (DAPI)/ml in 3\% BSA in PBS for $30 \mathrm{~min}$, mounted onto slides with $\mathrm{Gel} /$ Mount (Biomeda catalog M01), and viewed with a $60 \times$ water immersion lens on a Nikon Diaphot 300 microscope. Images were captured with a Hamamatsu charge-coupled device camera and Openlab software. All other cells were blocked in 3\% BSA-PBS; incubated with a 1:500 dilution of anti-GST monoclonal antibody (MAb; Santa Cruz catalog SC138), a 1:200 dilution of anti-Ran or 1:200 dilution of anti-RanBP1 polyclonal antibody (Santa Cruz catalog SC1159) as primary antibody, and Texas red-conjugated anti-mouse immunoglobulin $\mathrm{G}$ (IgG), Texas red-conjugated anti-goat IgG, or FITC-conjugated anti-rabbit IgG (all from Jackson ImmunoResearch Laboratories, diluted 1:500), with DAPI (10 $\mathrm{ng} / \mathrm{ml}$ ); and mounted and viewed as described above. 
Anti-BP1 IF
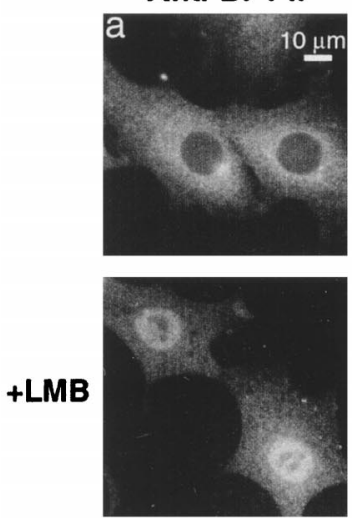

DAPI
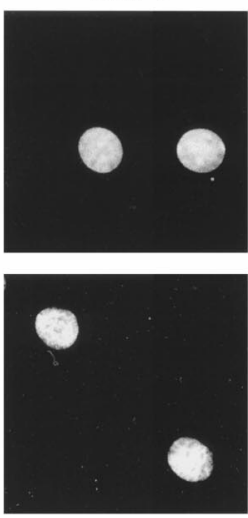

GFP-BP1
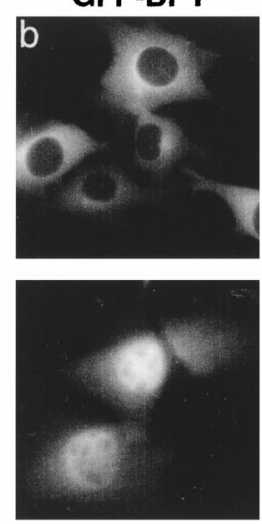

DAPI
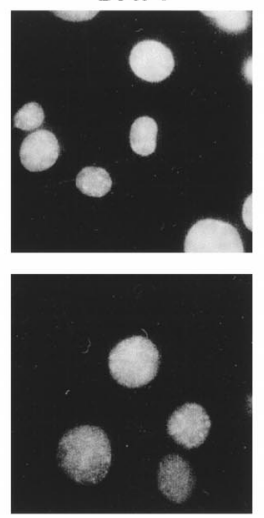

GFP-GFP-BP1
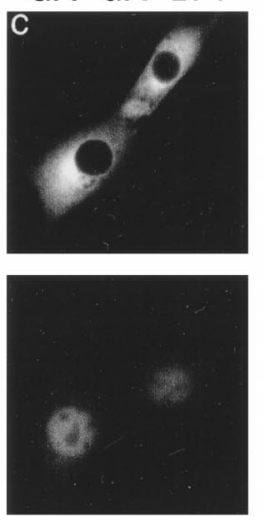

DAPI
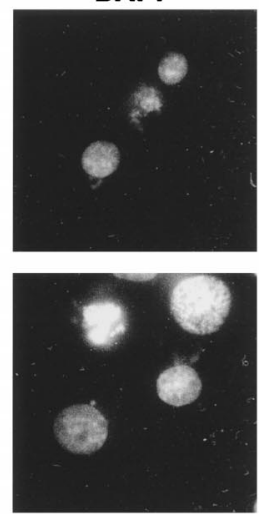

FIG. 1. RanBP1 is able to accumulate in the nucleus in the presence of LMB. BHK-21 cells were either untransfected (a panels) or transfected with pKGFPRanBP1 (b panels) or pKGFP-GFP-RanBP1 (c panels); 72 h posttransfection, cells were either untreated or incubated with $200 \mathrm{nM}$ LMB for 30 min at $37^{\circ} \mathrm{C}$. Cells expressing GFP fusion proteins were fixed, permeabilized, and stained with DAPI. Untransfected cell cultures were fixed, permeabilized, and stained with anti-RanBP1 and Texas red-conjugated secondary antibody as described in Materials and Methods.

Affinity binding assays and immunoblots. Recombinant GST-importin $\beta$ (165 pmol) was bound to GSH-Sepharose beads $(55 \mu \mathrm{l})$ and incubated with RanBP1 (150 pmol) and Ran:GTP (150 pmol) for $90 \mathrm{~min}$ at $4^{\circ} \mathrm{C}$, to form a ternary complex. After being washed with $\mathrm{AB}$, the beads were divided into four aliquots and incubated with $50 \mathrm{pmol}$ of Crm1 and/or $50 \mathrm{pmol}$ of Ran:GTP in a 12- $\mu \mathrm{l}$ volume for $1 \mathrm{~h}$ on ice. The supernatants and beads were separated by brief centrifugation. The beads were washed once in AB. Proteins were denatured by boiling with sodium dodecyl sulfate-polyacrylamide gel electrophoresis (SDSPAGE) buffer and separated by electrophoresis on SDS-12\% gels. After transfer to nitrocellulose, the proteins were detected by immunoblotting with anti-Crm1 $(1: 1,000)$, anti-RanBP1 (1:200), or anti-Ran $(1: 2,500)$ antibody and with horseradish peroxidase-coupled secondary antibodies. Detection was by enhanced chemiluminescence.

Recombinant GST-RanBP1 wild type or mutants (20 pmol) were bound to GSH-Sepharose $(40 \mu \mathrm{l})$ and incubated with 20 pmol each of Ran:GTP and importin $\beta$ at $4^{\circ} \mathrm{C}$ for $90 \mathrm{~min}$ at $1,400 \mathrm{rpm}$. Beads were then washed twice with Ran binding buffer (20 mM MOPS [pH 7.1], $100 \mathrm{mM}$ potassium acetate, $5 \mathrm{mM}$ magnesium acetate, $5 \mathrm{mM}$ dithiothreitol, $0.5 \%$ BSA, $0.05 \%$ Tween 20 ). Beadbound proteins were treated as above, immunoblotted with anti-Ran $(1: 2,500)$ and anti-importin $\beta(1: 2,000)$ or anti-GST $(1: 4,000)$ with HRP-horseradish peroxidase-conjugated anti-mouse IgG, and detected by enhanced chemiluminescence. Nucleoporins were immunoblotted with MAb 414 (BAbCo, Richmond, Calif.), and importin $\beta$ was detected using an antibody from Transduction Laboratories.

\section{RESULTS}

Inhibition of nuclear export results in the nuclear accumulation of RanBP1 and GFP-RanBP1. LMB can bind to the karyopherin family member, Crm1, and specifically inhibit export from the nucleus of proteins that contain leucine-rich NES sequences $(21,22,34,44)$. RanBP1 contains such an NES near its $\mathrm{C}$ terminus $(53,68)$. If RanBP1 shuttles constitutively between the nuclear and cytoplasmic compartments, LMB should inhibit its export but not its import and cause nuclear accumulation of the protein. However, if during interphase RanBP1 were retained in the cytosol by association with a constitutively cytosolic protein, LMB would have no effect on the subcellular location of RanBP1.

We tested these alternate hypotheses in two ways. First, BHK cells were incubated for 30 min with or without $200 \mathrm{nM}$ LMB and then fixed and stained for endogenous RanBP1. The RanBP1 in control cells was localized exclusively to the cytosol. LMB treatment led to a substantial redistribution into the nuclei (Fig. 1a). As a second approach, BHK cells were transiently transfected with a vector encoding GFP-RanBP1. Treatment with LMB led to a significant redistribution of the GFP fluorescent signal from the cytosol to the nucleus (Fig. 1b). Importantly, this effect was not a nonspecific result of
LMB treatment, which under similar conditions (60-min preincubation) did not inhibit import of a microinjected NLS cargo, GST-GFP-NLS (data not shown). Thus, inhibition of Crm1-mediated nuclear protein export results in the accumulation of RanBP1 within the nucleus. Moreover, the size of the GFP-RanBP1 fusion protein (approximately $50 \mathrm{kDa}$ ) is large enough that its nuclear import is unlikely to occur by simple diffusion within the time frame of the experiment. To confirm that import is not diffusive, we constructed a GFP-GFPRanBP1 fusion protein (approximately $75 \mathrm{kDa}$ ), which also redistributed into the nuclei of cells treated as above with LMB (Fig. 1c).

Low temperatures inhibit RanBP1 nuclear import. The estimated upper bound for the passive diffusion of proteins through the nuclear pores has been estimated to be about $50 \mathrm{kDa}$. Therefore, RanBP1 at $23 \mathrm{kDa}$ is in principle small enough to diffuse into the nucleus. However, other proteins of similar size, such as ribosomal proteins, histones, and Ran, have been found to be transported as complexes with other factors $(9,33,51,59)$. Passive diffusion rates vary linearly with the absolute temperature and so are relatively insensitive to the effect of cooling on ice. Therefore, we tested whether chilling would abrogate the nuclear accumulation of RanBP1 in the absence or presence of LMB. BHK-21 cells were cooled on ice for $30 \mathrm{~min}$ and then incubated with or without $200 \mathrm{nM}$ LMB for a further $30 \mathrm{~min}$ on ice. LMB did not cause nuclear accumulation of endogenous RanBP1 (Fig. 2b) or of GFPRanBP1 (Fig. 2d) under these conditions, suggesting that RanBP1 import does not occur by a process of passive diffusion through the nuclear pores. In interpreting these data, it should be appreciated that chilling the cells will reduce the rates of both Ran:GTP formation and consumption. Therefore, inhibition of RanBP1 import by cooling is unlikely to result from reduced levels of nuclear Ran:GTP. In this context, it is also important to note that chilling to $4^{\circ} \mathrm{C}$ completely inhibits export of a microinjected GST-GFP-NES cargo (data not shown) so that even in the absence of LMB, RanBP1 that entered the nuclei by simple diffusion would not be reexported.

Collapse of the Ran gradient causes equilibration of RanBP1 across the nuclear envelope. The nucleocytoplasmic Ran:GTP gradient is essential for many types of traffic through the nuclear pores, including protein import and export. Collapse of the Ran gradient might therefore be predicted to curtail RanBP1 export and permit its accumulation in the 

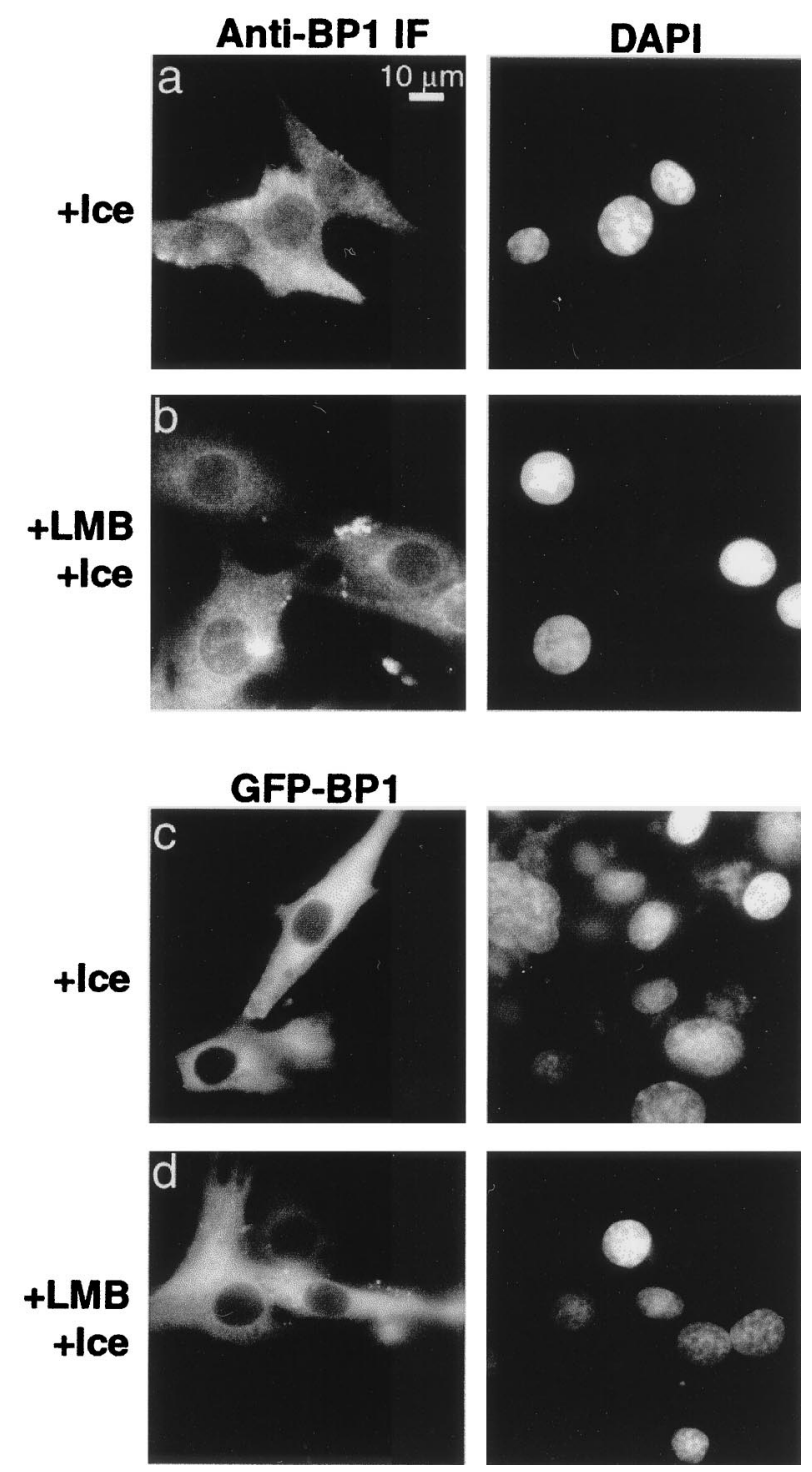

FIG. 2. Nuclear import of RanBP1 is inhibited by incubation on ice. BHK-21 cells were either mock transfected ( $\mathrm{a}$ and $\mathrm{b}$ ) or transfected with pKGFP-RanBP1 (c and d); $72 \mathrm{~h}$ posttransfection, cells were either incubated on ice for $1 \mathrm{~h}$ or incubated on ice for $30 \mathrm{~min}$ and then treated with $200 \mathrm{nM}$ LMB for an additional $30 \mathrm{~min}$ on ice. Cells expressing GFP fusion proteins were stained with DAPI. Mock-transfected cell cultures were stained with anti-RanBP1 and Texas redconjugated secondary antibody as described in Materials and Methods.

nucleus. However, if Ran:GTP were required for import, the loss of nuclear Ran:GTP could conceivably prevent such accumulation. To distinguish between these possibilities, we collapsed the Ran:GTP gradient by microinjecting nuclei of BHK cells with recombinant RanGAP. At high concentrations, the GAP can destroy Ran:GTP faster than it is produced by RCC1 and thus inhibit nuclear protein export (32). As shown in Fig. $3 \mathrm{~b}$, this treatment also resulted in the equilibration of RanBP1 across the nuclear envelope. Recombinant Ran, injected as a negative control, had no effect on RanBP1 localization (Fig. 3a) and demonstrated that the nuclear fluorescence in cells injected with RanGAP is not a result of bleed-through into the red channel of emission from the FITC-dextran marker. Together with the previous results, these data confirm that RanBP1 can shuttle between the cytoplasmic and nuclear com- partments and that the import of RanBP1 is Ran independent while export is Ran dependent.

Microinjected GST-RanBP1 lacking an NES can accumulate in the nucleus. The previous experiments to demonstrate nuclear import of RanBP1 depended on perturbations in normal cell function-either inhibition of the protein export pathway or collapse of the Ran gradient. One might argue, therefore, that RanBP1 does not usually enter the nucleus but is induced to do so only in response to a disruption in the normal nucleocytoplasmic traffic. To test this possibility, we microinjected into the cytosol of BHK-21 cells a GST-RanBP1(1-161) fusion protein that contains an intact RanBD but lacks the C-terminal NES (53). Within $15 \mathrm{~min}$, accumulation of this protein was visible within the nuclei of a significant fraction of the injected cells (Fig. 4).

Therefore, the RanBP1 RanBD is capable of nuclear entry and accumulation even in cells in which nuclear transport has not been perturbed. Taken together with results of the other experiments described above, these data suggest that nuclear import of RanBP1 is an ongoing process in interphase cells and that the steady-state cytoplasmic distribution of RanBP1 requires active export.

Curiously, the rate of import of GST-RanBP1(1-161) was not uniform. Within a group of cells injected within several seconds of one another, there were often single cells that did not accumulate the fusion protein within their nuclei, at least within the short incubation times permitted (e.g., Fig. 4, top panel). The reason for this heterogeneity of response is unclear at present.

Nuclear accumulation of RanBP1 requires energy and soluble factors. To determine the mechanism by which RanBP1 enters the nucleus, we used digitonin-permeabilized cells that were competent for nuclear protein transport. As a substrate we used GST-RanBP1(1-161). After incubation with the cells, the fusion protein was detected by immunofluorescence with anti-GST antibodies. GST-RanBP1(1-161), either alone or with an ATP/GTP-regenerating system, did not accumulate in the nuclei (Fig. 5A, panel a). Similarly, the addition of reticulocyte lysate as a source of soluble factors did not, in the absence of energy, lead to accumulation of the RanBP1 construct (panel b). However, rapid accumulation did occur when both reticulocyte lysate and energy were provided (panel c). A similar result was obtained when full-length GST-RanBP1 was used in this assay, in the presence of LMB to inhibit export, although some nuclear accumulation of the GST-RanBP1 was detectable even in the absence of energy (data not shown).

These data indicate that the nuclear accumulation of RanBP1 is an active process. However, in vitro nuclear transport assays depend critically on the integrity of the nuclear envelope. If the envelope is damaged and the import substrate binds to insoluble nuclear components, then false positives can arise. To validate our import assay, we added GST-RanBP1 (1-161), plus energy and reticulocyte lysate, to cells that had been deliberately damaged by addition of $0.2 \%$ Triton X-100, which dissolves the nuclear envelope. As shown in Fig. 5B (panels a and b), this treatment did not result in the binding of the RanBP1 to insoluble nuclear components. Second, we tested whether the nuclei of the digitonin-permeabilized cells were accessible to antibodies by performing an import assay using GST-RanBP1(1-161) as described above except that after fixation, the membranes were not dissolved with methanol. Under these conditions, the anti-GST antibody could not gain access to the nuclei to stain the substrate (panel c), whereas staining was efficient when the cells were treated with methanol (panel a). These tests demonstrate that the digitonin-permeabilized cells used in the assay possess intact nuclear enve- 
FITC-Dextran
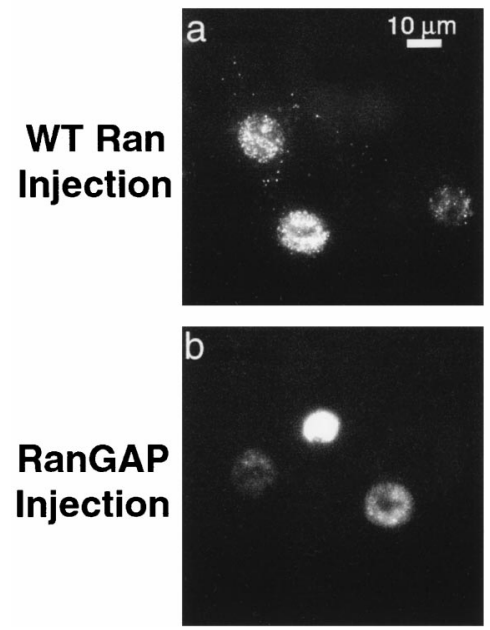

FIG. 3. Collapse of the nucleocytoplasmic Ran gradient causes an equilibration of RanBP1 across the nuclear envelope. The nuclei of BHK-21 cells were microinjected with FITC-dextran $(0.2 \mathrm{mg} / \mathrm{ml})$ as a site-of-injection marker (left) along with either wild-type (WT) Ran $(1.0 \mathrm{mg} / \mathrm{ml}$; a panels) or RanGAP $(0.4$ $\mathrm{mg} / \mathrm{ml}$; b panels) and incubated for $10 \mathrm{~min}$ at $37^{\circ} \mathrm{C}$. The intracellular distribution of endogenous RanBP1 was assessed by indirect immunofluorescence (IF) with anti-RanBP1 and Texas red-conjugated to anti-goat IgG (right) as described in Materials and Methods. Arrows are drawn to help distinguish injected cells from uninjected cells.

lopes and that damage to the envelopes does not result in a false positive.

To determine which soluble factors are required for nuclear accumulation of the GST-RanBP1(1-161), the fusion protein was added to permeabilized cells together with various combinations of recombinant transport factors. As shown in Fig. $6 \mathrm{~A}$, the addition of Ran plus an energy source led to a small amount of nuclear accumulation (panel e), which was substantially increased by the addition of the transport receptor, importin $\beta$ (panel h). There are at least two possible interpretations of this result. First, RanBP1 may be imported like a more classical transport substrate, in a complex with importin $\beta$ and Ran:GDP (12). Second, RanBP1 may translocate across the nuclear pores by itself, or with Ran, and accumulate in the nucleus as a ternary complex with Ran:GTP and importin $\beta$.

To begin to distinguish between these possibilities, we tested whether the Ran exchange factor, RCC1, could stimulate RanBP1 import in the absence of exogenous importin- $\beta$ (Fig. $6 \mathrm{~A}$, panel i). We chose RCC1 because it is rapidly accumulated within nuclei (M. E. Nemergut and I. G. Macara, submitted for publication), where it efficiently drives the formation of Ran: GTP. It does not bind RanBP1 directly, although RanBP1 can inhibit catalysis of nucleotide exchange by RCC1 (6). Interestingly, the RCC1 did significantly elevate the nuclear accumulation of GST-RanBP1(1-161) (panel i). Neither importin $\beta$ nor RCC1 in the absence of Ran was able to mediate import to a significant level (panels $\mathrm{f}$ and $\mathrm{g}$ ). Note that during preparation, the permeabilized cells are depleted of the majority of their endogenous importin $\beta$, compared to the level of nucleoporins detected by MAb 414 (Fig. 6B), and the residual importin $\beta$ is insufficient to drive the nuclear accumulation of a classical NLS (data not shown). Therefore, nuclear accumulation of RanBP1 in vitro may require Ran:GTP, but the addition of a karyopherin such as importin $\beta$ is not essential (although importin- $\beta$ can enhance accumulation).

Taken together, these results suggest that RanBP1 can enter the nucleus either as a complex with Ran or by a factorindependent mechanism and is then sequestered within the nucleus by binding Ran:GTP or by the formation of a ternary complex with Ran and importin $\beta$ (or other karyopherins).

RanBP1 accumulation in the nucleus requires Ran:GTP. To further distinguish between these possibilities, we tested whether import of GST-RanBP1(1-161) was inhibitable by dominant interfering mutants of Ran and importin $\beta$ or wheat germ agglutinin (WGA), each of which blocks classical, receptor-mediated transport through the nuclear pores. WGA and the dominant interfering mutant of importin $\beta$ both substantially reduced the extent of import (Fig. 7A panels $h$ and $j$ ), and consistent with previous results, the removal of energy by addition of apyrase also prevented accumulation of the GSTRanBP1(1-161), indicating that it is an active process (panel g).

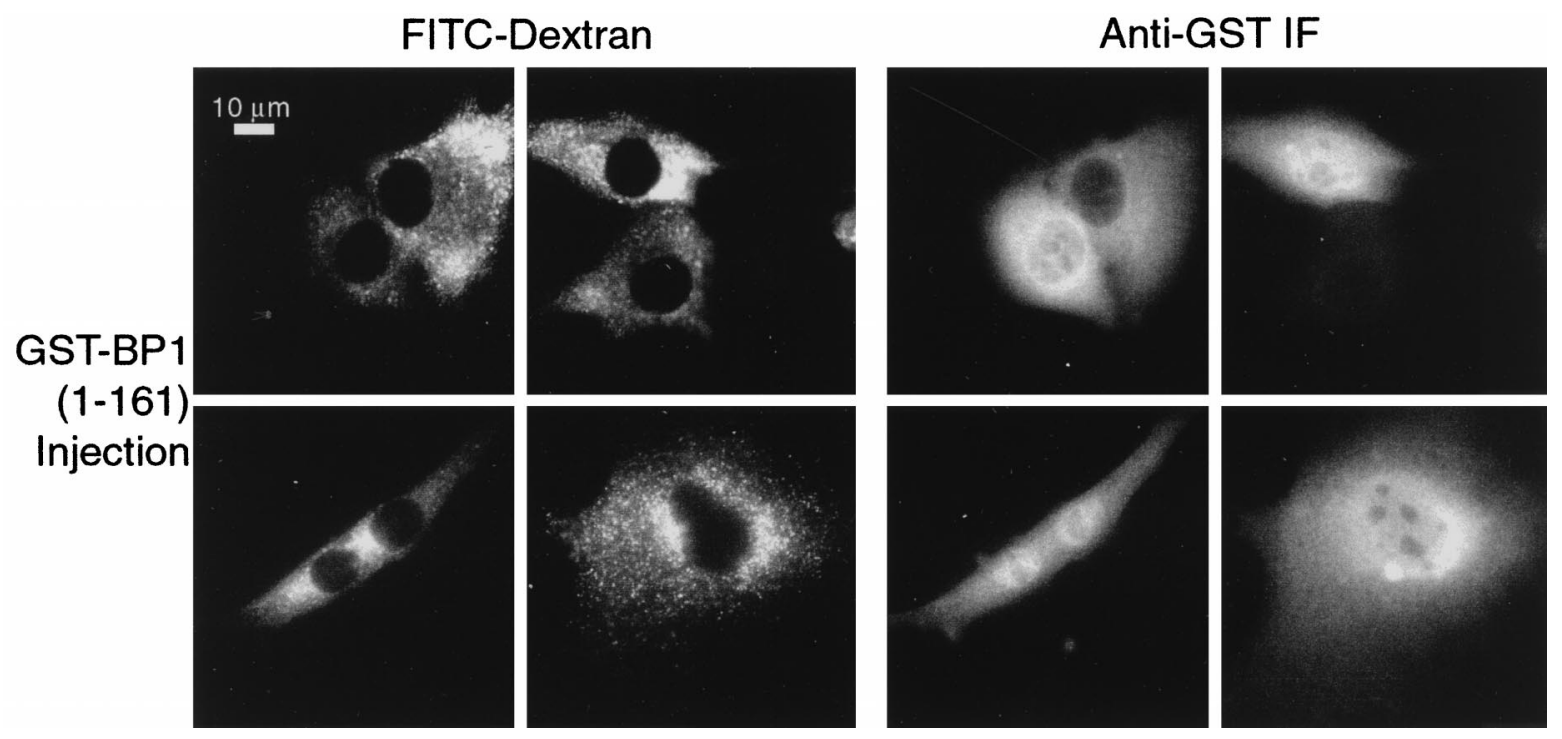

FIG. 4. GST-RanBP1(1-161) is imported into the nucleus. The C-terminal truncation mutant of RanBP1, which lacks the NES, was expressed as a GST fusion protein and injected at a concentration of $1.0 \mathrm{mg} / \mathrm{ml}$ into the cytosol of BHK-21 cells, together with FITC-dextran $(2.0 \mathrm{mg} / \mathrm{ml}) \mathrm{as}$ an injection site marker. After $15 \mathrm{~min}$ incubation at $37^{\circ} \mathrm{C}$, the cells were fixed and stained for GST. A gallery of injected cells is shown. 

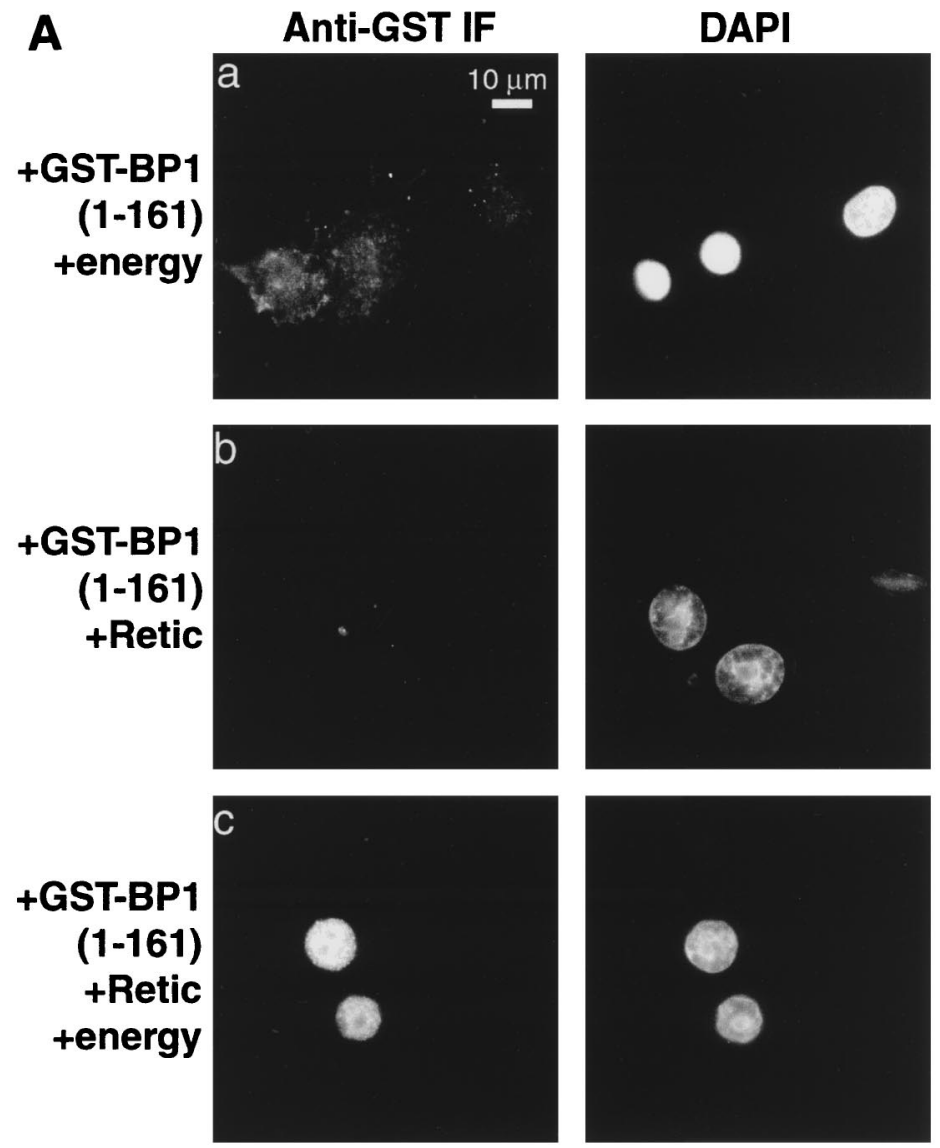

B
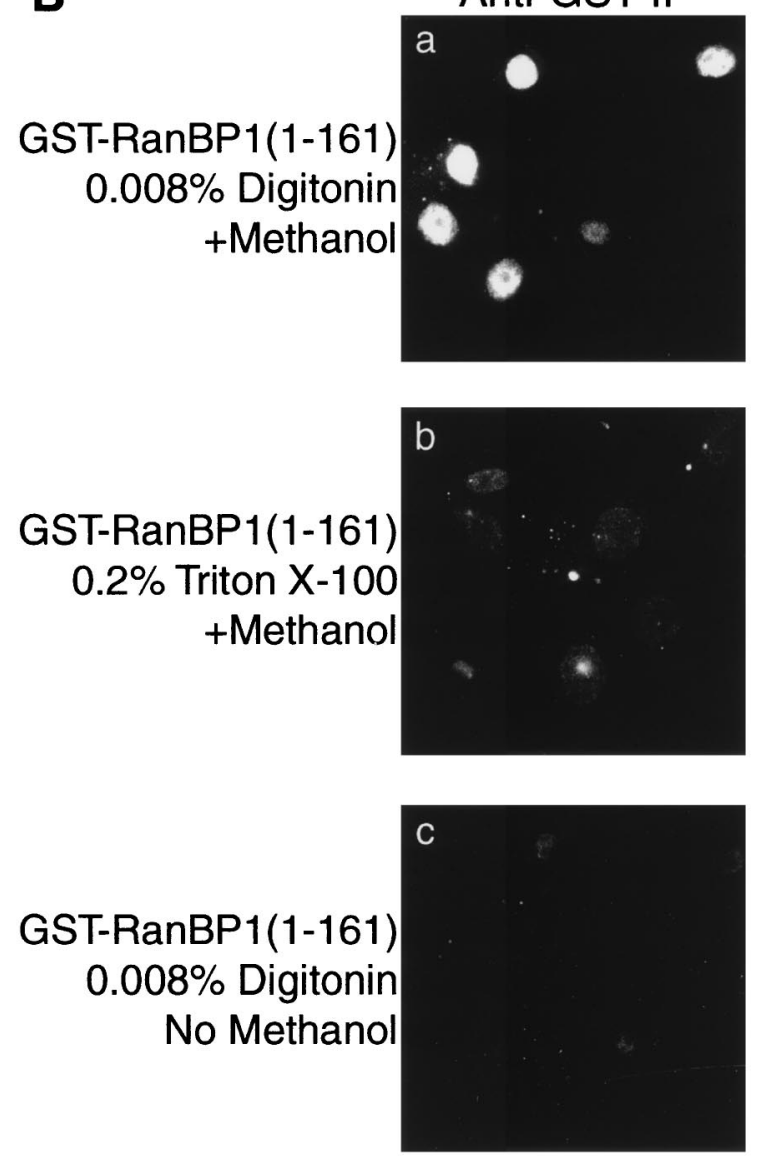

FIG. 5. Nuclear accumulation of GST-RanBP1(1-161) requires addition of energy and soluble factors. (A) BHK-21 cells were permeabilized with 0.008\% digitonin and overlaid with $3 \mu \mathrm{M}$ GST-RanBP1(1-161) and either an ATP/GTP-regenerating system (a), reticulocyte lysate (Retic; b), or reticulocyte lysate and ATP/GTPregenerating system (c). The cells were incubated at room temperature for $30 \mathrm{~min}$ and then fixed and stained for GST. Nuclei were stained with DAPI (right). (B) Import of GST-RanBP1(1-161) is not an artifact of damaged nuclear envelopes. Import was performed as for panel A, using either digitonin-permeabilized (a and c) or Triton X-100-extracted (b) cells. Following fixation, the cells were either incubated directly with anti-GST antibody (c) or first incubated with methanol ( $\left.-20^{\circ} \mathrm{C}\right)$ to dissolve the nuclear membrane ( $\mathrm{a}$ and $\mathrm{b}$ ).

However, the addition of Ran(G19V) had no inhibitory effect, although it completely blocked import of the control substrate, GST-GFP-NLS (Fig. 7A, panel i; Fig. 7B). This result suggests that RanBP1 does not enter the nucleus as a complex with Ran:GDP and importin $\beta$.

It is important to note that WGA and importin $\beta(45-462)$ also block Ran import $(51,59)$ and do not, therefore, distinguish whether the observed inhibition is of RanBP1 translocation across the nuclear pores or of RanBP1 accumulation within the nucleus. It is also of note that although Ran normally enters the nucleus as a complex with GDP, the Ran(G19V) mutant, which is predominantly GTP bound, can accumulate both within the nucleoplasm and at the nuclear pores (39).

Taken together, these data support the idea that Ran:GTP is required for nuclear accumulation of GST-RanBP1(1-161). To test this hypothesis further, we performed in vitro import assays using various concentrations of Ran at a fixed concentration of GST-RanBP1(1-161) and RCC1. After fixation, the cells were stained for both GST and Ran. We observed that the fluorescence intensity of the nuclear GST-RanBP1(1-161) correlated quite well with the nuclear Ran fluorescence (Fig. 8A). We then quantitated the relative nuclear fluorescence levels of GST-RanBP1(1-161) in individual cells and plotted the mean values against the Ran concentration added to the cells (Fig. 8B). The GST-RanBP1(1-161) levels show a strong linear correlation with the Ran concentration (linear correlation coefficient $[r]=0.967)$. The most likely explanation of this result is that GST-RanBP1(1-161) enters the nucleus alone, by a process of facilitated diffusion, and that association with Ran:GTP within the nucleus prevents its export. Alternatively, the RanBP1 may enter as a complex with Ran:GTP, and become trapped within the nucleus by association with other factors.

A RanBP1 mutant is defective in nuclear import. To distinguish between these two hypotheses, we examined a number of RanBP1 variants that we had previously generated using a random mutagenesis approach (47). We chose two mutants that each exhibit a substantially reduced affinity for Ran:GTP (Fig. 9A). One mutant contains a single residue substitution, $\mathrm{E} 37 \mathrm{~K}$, and the other is a double mutant, G71C/K76E. Both mutants possess the unusual property that they can form stable ternary complexes with Ran:GTP plus either importin 5 (47) or importin $\beta$ (Fig. 9B).

The two mutants were expressed ectopically as GFP fusions in BHK-21 cells, and their ability to enter the nucleus was tested by the addition of LMB, to block export. In the absence of LMB, both mutants localized exclusively to the cytosol (Fig. 


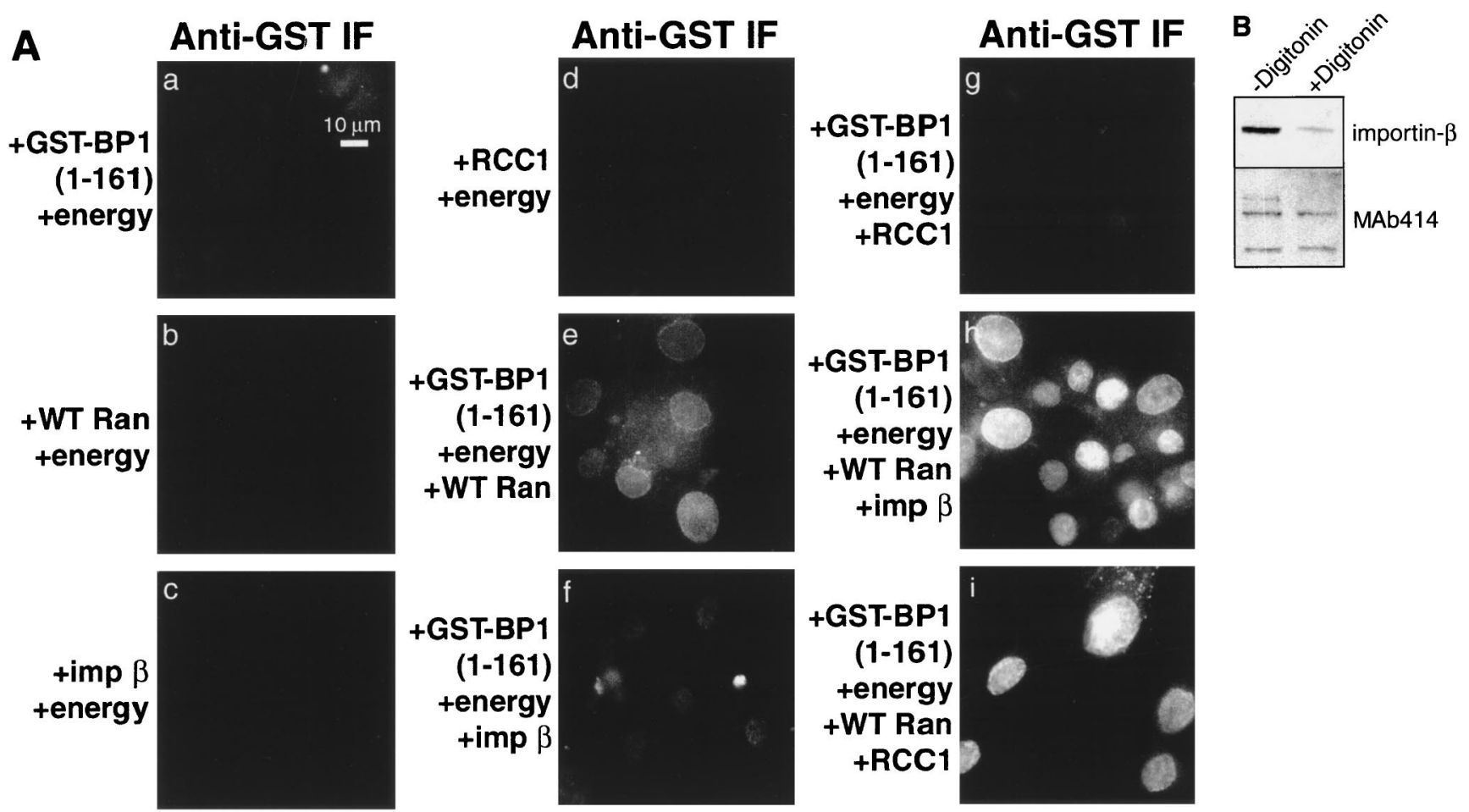

FIG. 6. Nuclear accumulation of GST-RanBP1(1-161) requires energy, Ran plus importin $\beta$, or RCC1. (A) BHK-21 cells were permeabilized with digitonin and overlaid with an energy-regenerating system and different combinations of the following factors: $3 \mu \mathrm{M}$ GST-RanBP1(1-161), $3 \mu \mathrm{M}$ wild-type (WT) Ran, $3 \mu \mathrm{M}$ importin (imp) $\beta$, and $3 \mu \mathrm{M}$ RCC1. Cells were incubated at room temperature for 30 min and then fixed and stained for GST. (B) Loss of importin $\beta$ from permeabilized cells. Cells were solubilized in SDS buffer either before or after permeabilization with digitonin, and samples were analyzed by SDS-PAGE followed by immunoblotting with antibodies against either importin $\beta$ or glycosylated nucleoporins (MAb 414).

9C, panels b and c). Surprisingly, however, they behaved differently in the presence of LMB. The G71C/K76E mutant accumulated within the nucleus to a degree similar to that of the wild-type protein, but the E37K mutant remained excluded from the nucleus (panels e and f). Importantly, at $4^{\circ} \mathrm{C}$ in the presence of LMB, the GFP-RanBP1(E37K) did not equilibrate across the nuclear envelope, as would be expected if accumulation, rather than transport, were defective (Fig. 9D, panel b). Additionally, a GST-RanBP1(1-161,E37K) recombinant protein was excluded from the nucleus when microinjected into the cytoplasm of BHK-21 cells (Fig. 9E), unlike the control GST-RanBP1(1-161) protein (Fig. 4). Four other mutants of RanBP1 that were tested all behaved like the G71E/K76E mutant and wild-type RanBP1, accumulating in the nuclei of LMB-treated cells (data not shown).

These results suggest that the translocation of RanBP1 into the nucleus most likely occurs in the absence of association with Ran:GTP and confirm that import occurs by a specific, facilitated pathway rather than by simple diffusion. The E37K mutation lies in the N-terminal arm of the RanBD, which embraces Ran:GTP, while the other mutations are located in different regions of the binding domain. Thus, these results implicate the N-terminal arm of the RanBD in the nuclear transport mechanism of RanBP1.

Crm1 dissociates RanBP1 from a RanBP1-Ran:GTP-importin $\boldsymbol{\beta}$ ternary complex. If RanBP1 can accumulate in the nucleus as a ternary complex with Ran:GTP and importin $\beta$, how is it efficiently exported back to the cytosol? One possibility is that Crm1 exports the entire complex; another is that Crm1 displaces importin $\beta$ from the complex and exports only the RanBP1. To distinguish between these different mechanisms, recombinant GST-importin $\beta$ was attached to GSH-Sepharose beads and mixed with Ran:GTP and RanBP1 to form a tethered ternary complex on the beads. After washing to remove free Ran and RanBP1, the beads were incubated either with buffer or with purified Crm1 (29). The beads and eluate were then analyzed by immunoblotting for Ran, RanBP1, and Crm1. As shown in Fig. 10, incubation with Crm1 led to release into the eluate of a substantial fraction of the RanBP1 and of the Ran from the GST-importin $\beta$. No Crm1 was found to be associated with the complex on the Sepharose beads. Therefore, we conclude that Crm1 promotes the dissociation of Ran and RanBP1 from importin $\beta$, or sequesters free Ran and RanBP1, which drives dissociation from the importin $\beta$ by mass action. In either case, the Crm1 appears unable to form a complex with Ran and RanBP1 that includes importin $\beta$.

\section{DISCUSSION}

One of the hallmarks of the nuclear transport machinery is the asymmetric distribution of its soluble components. The Ran:GTP gradient across the nuclear pores is critical to many forms of protein and nucleic acid transport, and the dissociation of transport complexes in the cytosol requires both RanGAP and RanBP1. It is therefore essential to understand how the asymmetric distribution of transport factors is initiated and maintained. Ran:GDP import has been demonstrated to be mediated by NTF2 $(51,59)$. Importin $\beta$ and transportin appear able to cross the nuclear pores in the absence of other factors $(35,43)$. Importin $\alpha$ is recycled back to the cytosol by CAS (37). We now present evidence that RanBP1 import also occurs via a specific, facilitated mechanism. 
A

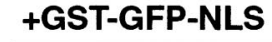

+ Retic +energy
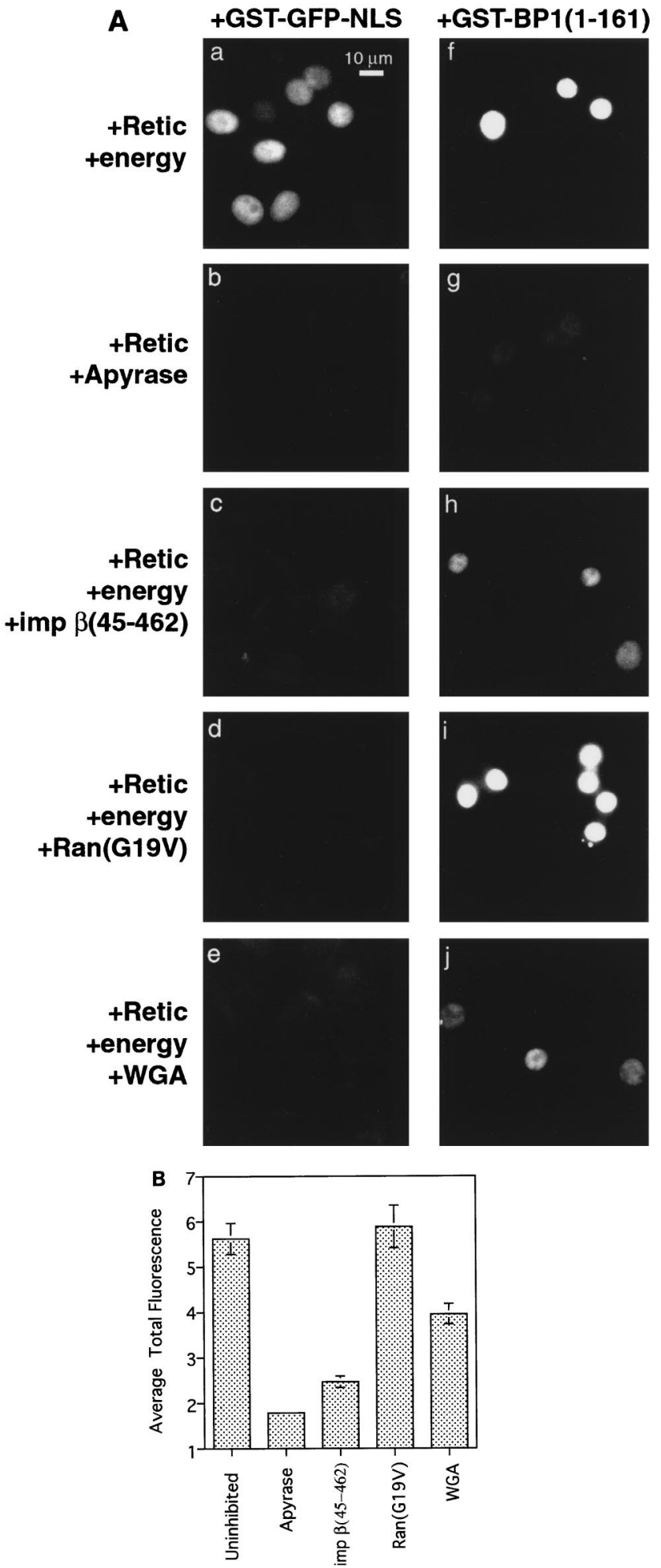

FIG. 7. Inhibitors of the NLS pathway do not inhibit GST-RanBP1(1-161) import. (A) BHK-21 cells were permeabilized with $0.008 \%$ digitonin and overlaid with either 1.5 $\mu \mathrm{M}$ GST-GFP-NLS (a to e) or $3 \mu \mathrm{M}$ GST-RanBP1(1-161) (f to j) in the presence of an energy-regenerating system, reticulocyte lysate (Retic), $100 \mathrm{U}$ of apyrase/ml, $2 \mu \mathrm{M}$ importin (imp) $\beta(45-462)$ ), $100 \mathrm{mg}$ of RanG19V/ml, or $200 \mathrm{mg}$ of WGA $/ \mathrm{ml}$. Cells were incubated at $23^{\circ} \mathrm{C}$ for $30 \mathrm{~min}$ and then fixed. GST-GFP-NLS and GST-RanBP1(1-161) were detected by epifluorescence and by indirect immunofluorescence with anti-GST MAb, respectively. (B) The average total fluorescence per cell of GST-RanBP1(1-161) was quantitated using Openlab software. Error bars represent the standard error of the mean $(n>20$ cells per treatment).
RanBP1 functions as a coactivator of RanGAP and thereby mediates the dissociation of complexes between Ran:GTP and transport receptors of the importin $\beta$ superfamily. This function requires that RanBP1 be localized to the cytosol. However, RanBP1 is small enough, in principle at least, to be able to enter the nucleus by passive diffusion (although the protein behaves in solution as a dimer [6]). Because RanBP1 binds with nanomolar affinity to Ran:GTP, which is concentrated within the nucleus, and can form a ternary complex with importin $\beta$, which is also predominantly nuclear, a mechanism must exist either to sequester the RanBP1 in the cytosol or to actively export it from the nucleus. RanBP1 does contain a leucine-rich NES near its $\mathrm{C}$ terminus but also contains adjacent sequences that may function in cytosolic retention (53).

We now show that RanBP1 shuttles constitutively between the cytosolic and nuclear compartments and that its cytosolic distribution is actively maintained by nuclear export. It is likely that this process is conserved through evolution, because Yrb1p, the yeast homologue of RanBP1, though lacking a C-terminal NES, has been shown to accumulate in the nucleus under conditions in which export is likely inhibited $(27,56)$. GST-RanBP1 lacking an NES accumulates in the nuclei both of intact cells when the protein is microinjected into the cytosol and of permeabilized cells in a Ran- and importin $\beta$-dependent fashion that requires energy.

Based on the observations that accumulation of RanBP1 in nuclei of intact cells was blocked by cooling the cells on ice, and that both GFP-GFP-RanBP1 ( 75 kDa) and GSTRanBP1(1-161) ( 50-kDa monomer; 100-kDa dimer) were capable of nuclear import, we consider it highly unlikely that RanBP1 transits the pores by simple diffusion. In vitro assays demonstrated that nuclear accumulation of GST-RanBP1(1$161)$ is active. Accumulation required energy and Ran. However, it is important to distinguish the mechanism of translocation through the pores from that of the net accumulation of a substrate within the nucleoplasm. These are entirely separate processes. Most import assays do not distinguish between them, however. A protein that can transit the pores by facilitated diffusion may accumulate against a concentration gradient if it binds to a nuclear protein or is covalently modified-for instance, by phosphorylation-within the nucleus in a manner that prevents reexport. If the nuclear protein partner is imported via a classical pathway, then in vitro assays will display a dependence on energy and soluble factors even though these are not required for the translocation of the substrate itself. In the present case, the in vitro assays showed a strong dependence on Ran:GTP. This factor could be supplied either as a constitutively GTP-bound mutant [Ran (G19V)] or by addition of wild-type Ran plus RCC1. Factors that enhance nuclear accumulation of Ran, such as importin $\beta$, also enhanced RanBP1 accumulation. Factors that inhibit Ran accumulation, such as WGA or a dominant interfering mutant of importin $\beta$, also inhibited RanBP1 accumulation. Therefore, these assays could not distinguish whether the GST-RanBP1 (1-161) transits the nuclear pores alone and becomes trapped within the nucleoplasm by binding to Ran:GTP or, alternatively, transits the pores in a complex with Ran:GTP.

We were able to distinguish between these two hypotheses, however, by the use of point mutants of RanBP1. We chose two mutants with impaired affinity for Ran:GTP, E37K and G71C/K76E (47). Importantly, both of these proteins, which contain altered residues within the RanBD, retain the ability to form stable ternary complexes with karyopherins such as importin $\beta$ plus Ran:GTP. Thus, if capable of entering the nucleus, they could in principle become trapped in such a ternary complex and accumulate within the nucleoplasm. When ex- 
A

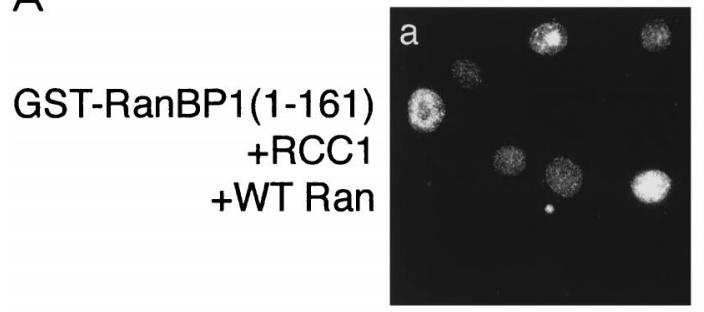

Anti-GST IF

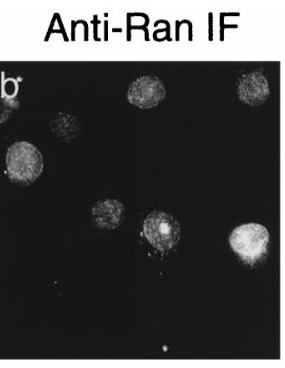

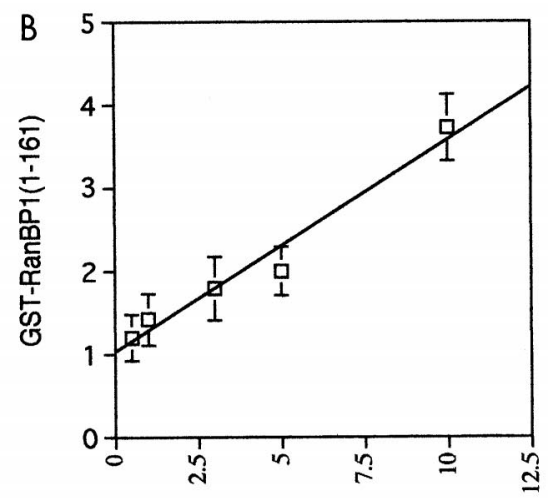

WT $\operatorname{Ran}(\mu \mathrm{M})$

FIG. 8. Nuclear accumulation of GST-RanBP1(1-161) is mediated by Ran:GTP. (A) Digitonin-permeabilized BHK-21 cells were incubated with Ran, RCC1, and an energy-regenerating system as for Fig. 6. GST-RanBP1(1-161) was detected using anti-GST and an anti-mouse conjugated to Texas red; Ran was detected with anti-Ran and an anti-rabbit antibody conjugated to FITC. WT, wild type. (B) Import assays were performed at a variety of Ran concentrations as in panel A. Images were captured; the average total fluorescence per cell of GST-RanBP1(1-161) was quantitated for different assays using Openlab software as described in Materials and Methods and plotted against the Ran concentration. Values are shown \pm standard error of the mean $(n=20)$. The line was drawn using a least squares algorithm. Correlation coefficient, $r=0.967$.

pressed in cells as a GFP fusion protein, the $\mathrm{G} 71 \mathrm{C} / \mathrm{K} 76 \mathrm{E}$ mutant accumulated within nuclei upon addition of LMB to block export. This result is consistent with a model in which RanBP1 transits the nuclear pores alone, not as a complex with Ran:GTP. Several other mutants with altered residues within the RanBD were also capable of nuclear import in the presence of LMB (data not shown). Additionally, Zolotukhin and Felber found that a mutant RanBP1 which is defective in Ran binding and lacks an NES could still accumulate in the nucleus of transfected cells (68). The ability of this mutant to form ternary complexes with Ran:GTP and importin $\beta$ was not tested, however, and so the mechanism of nuclear accumulation remains to be determined in this case.

Interestingly, we discovered one mutant, E37K, that was incapable of nuclear import under the conditions of the assay and did not even equilibrate across the nuclear envelope when the cells were held at $4^{\circ} \mathrm{C}$. This result confirms that RanBP1 cannot enter the nucleus by passive diffusion but must use a facilitated mechanism. The E37K mutation lies in the N-terminal arm of the RanBD, and the side chain faces outward into the solvent (62). Therefore, the unique transport defect of RanBP1(E37K) suggests that the N-terminal arm of the protein may be involved in nuclear import, perhaps by binding to nuclear pore components. In an independent study, a different group has identified another mutation within the RanBP1 of budding yeast (Yrb1p) that is unable to accumulate in nuclei when export is inhibited (Ed Hurt, personal communication). Their data support our conclusion that RanBP1 import does not occur by simple diffusion and that its nuclear accumulation is energy dependent.

Within the nucleoplasm, RanBP1 probably forms a ternary complex with Ran:GTP and karyopherins such as importin $\beta$ or other binding proteins. We therefore asked how the RanBP1 could be efficiently exported. We found that Crm1 cannot bind with measurable affinity to the RanBP1-Ran-importin $\beta$ ternary complex, but it facilitates the dissociation of RanBP1 and Ran from the complex. This mechanism may be required to prevent the RanBP1 from being sequestered within the nucleus.

Based on the data described above, we propose that RanBP1 is continually cycling between the cytosol and nucleus in interphase cells and that import occurs via a specific, nondiffusive pathway that does not require soluble factors such as the karyopherins and that involves the N-terminal arm of the RanBD. Accumulation within the nucleus against a concentration gradient can occur if the NES of RanBP1 is deleted or if export is inactivated. Accumulation is driven by binding to nuclear Ran: GTP, and most likely by the formation of ternary complexes with Ran:GTP and karyopherins. Endogenous RanBP1, however, binds to the exportin, Crm1, which prevents association with other karyopherins. Rapid export of the RanBP1 by Crm1 terminates the transport cycle.

The purpose of RanBP1 shuttling remains to be further investigated. However, we speculate that RanBP1 import may help clear the nuclear pores of Ran:GTP. Ran is sufficiently small that in principle it could diffuse through the pores $(55$, 62). Indeed, NTF2 (the nuclear import receptor for Ran:GDP) is not required for survival of yeast if the expression of Ran is elevated, and the requirement for NTF2 in nuclear import assays performed on permeabilized cells can be eliminated by the inclusion of a high Ran concentration in the assay buffer (45). Ran:GTP possesses an average molecular size that is very similar to that of Ran:GDP. Therefore Ran:GTP is expected to slowly diffuse out through the nuclear pores at about the same rate that it can diffuse in. Incoming nuclear transport complexes will be dissociated by interaction with any molecule of Ran:GTP that is encountered within the pore, and such a premature dissociation would most likely cause termination of the translocation process. By sweeping the pores free of Ran: GTP, RanBP1 may reduce the likelihood that this process occurs.

\section{ACKNOWLEDGMENTS}

We thank Bryce Paschal and Jim Holaska (University of Virginia) for the kind gifts of purified Crm1 protein and anti-Crm1 antiserum, Steve Adam (Northwestern University) for the importin $\beta$ cDNA, and Barbara Wolff-Winiski (Novartis) for the LMB.

This work was supported by grant GM 50526 from the National Institutes of Health, DHHS.

\section{REFERENCES}

1. Adam, S. A., R. Sterne-Marr, and L. Gerace. 1992. Nuclear protein import using digitonin-permeabilized cells. Methods Enzymol. 219:97-110.

2. Askjaer, P., T. H. Jensen, J. Nilsson, L. Englmeier, and J. Kjems. 1998. The specificity of the CRM1-Rev nuclear export signal interaction is mediated by 
A

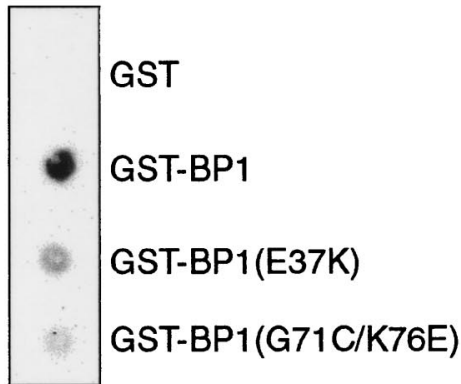

C

GFP-BP1
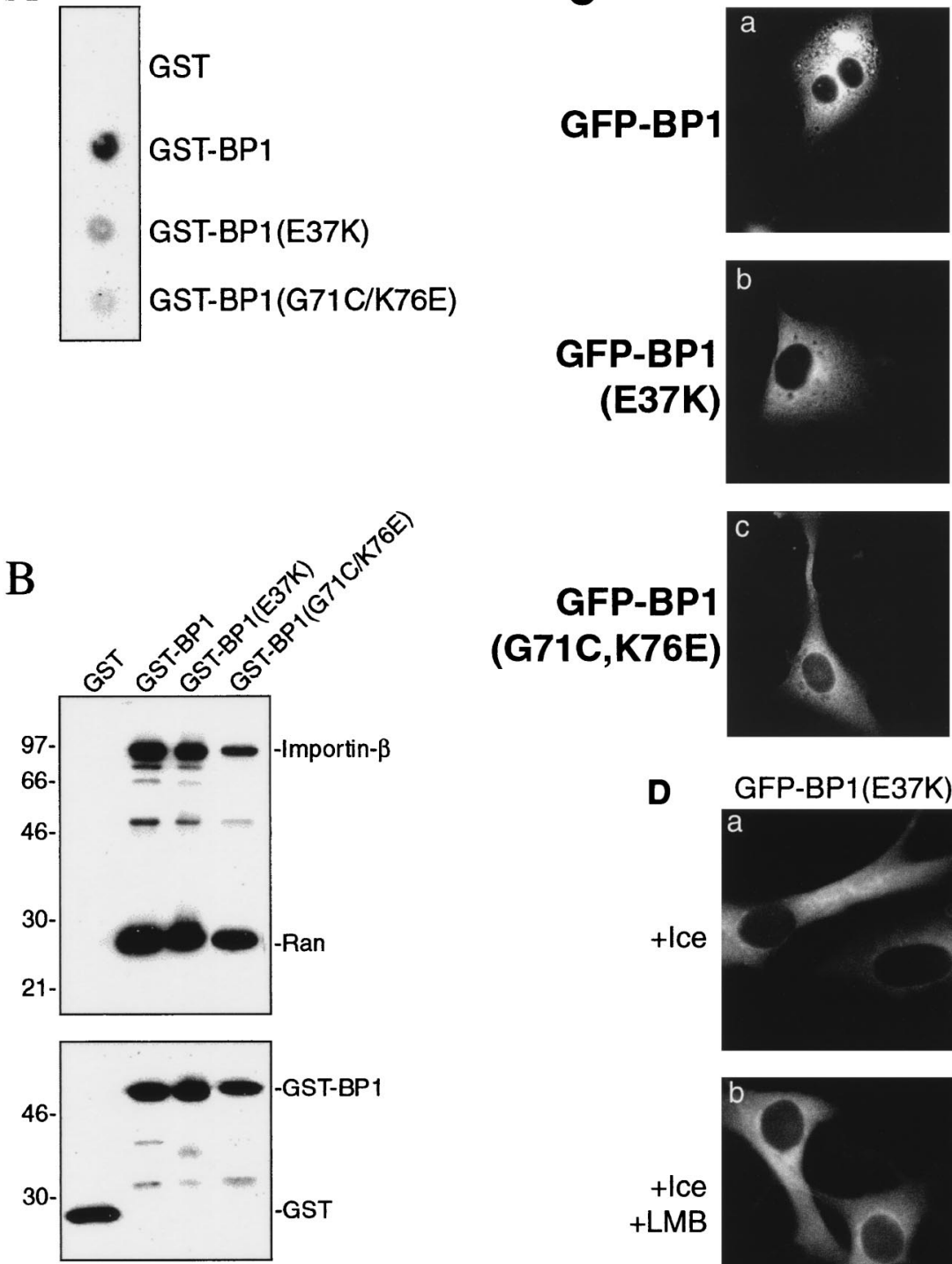

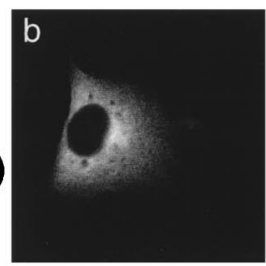

GFP-BP1

(E37K)

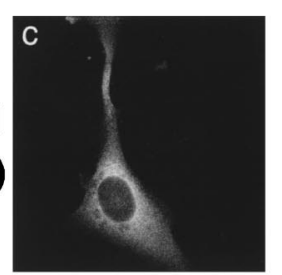

GFP-BP1 (G71C,K76E)

D
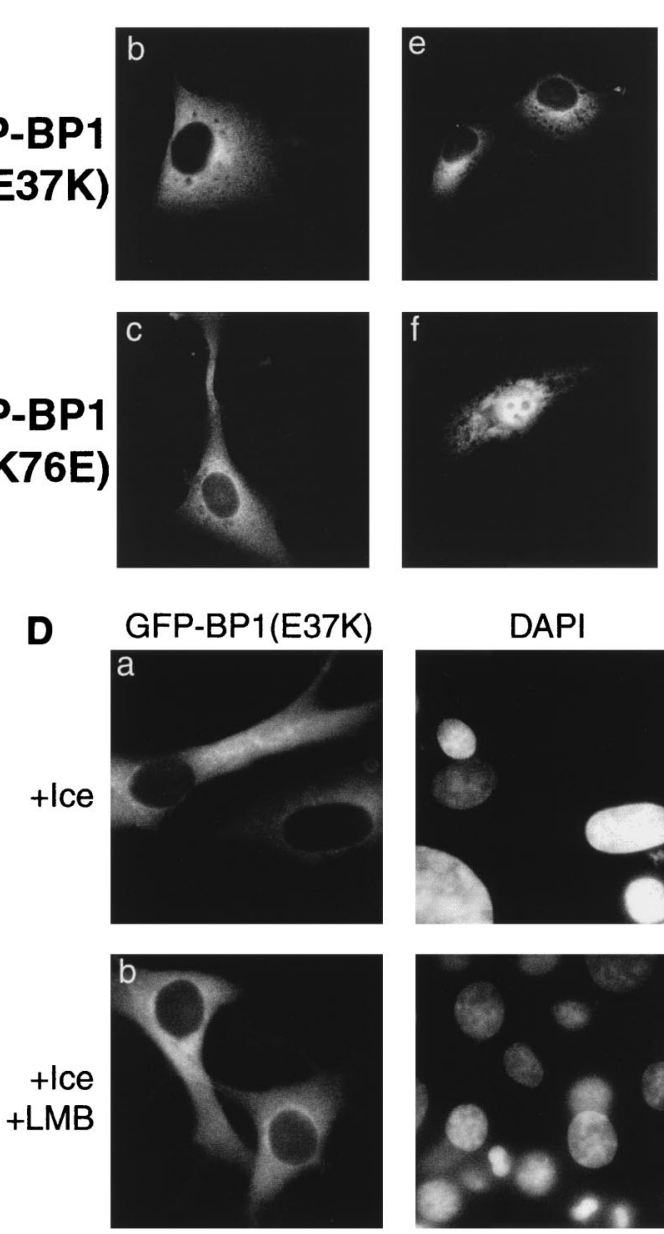

E
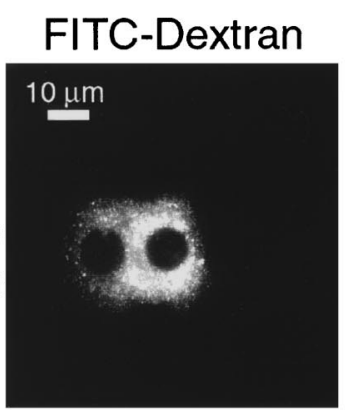
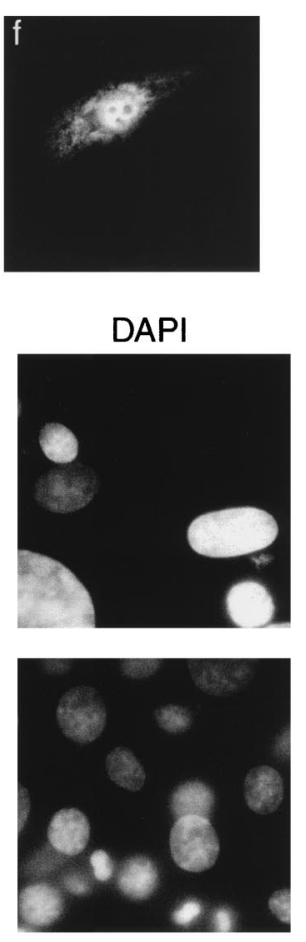

+LMB
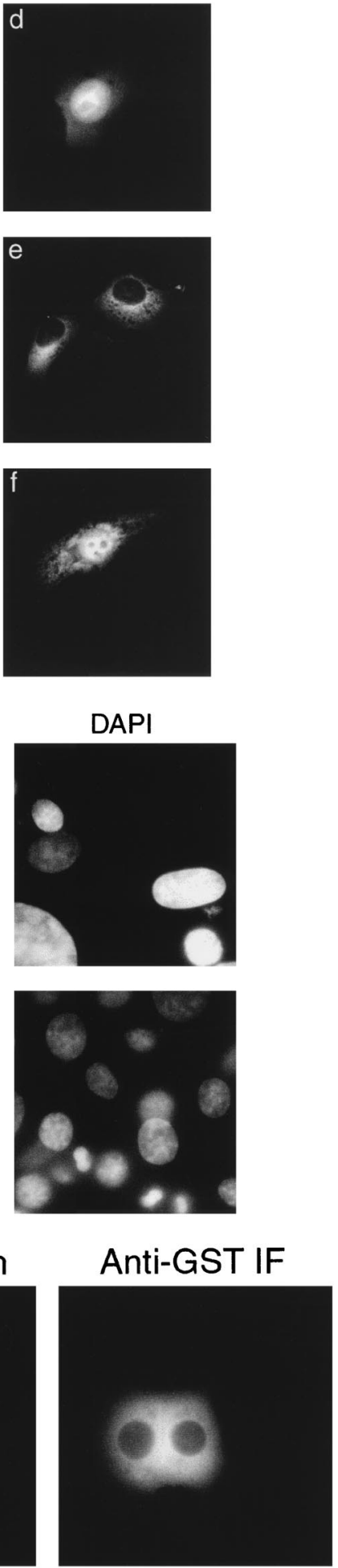

FIG. 9. An E37K mutant of RanBP1 does not import into nuclei but can form a ternary complex with Ran:GTP and importin $\beta$. (A) Equimolar amounts of recombinant wild-type or mutant GST-RanBP1s were spotted onto nitrocellulose and incubated with Ran ( $8 \mathrm{nM})$ loaded with $\left[\gamma^{-32} \mathrm{P}\right] \mathrm{GTP}$ for 30 min as described in Materials and Methods, washed, and exposed to film with an intensifying screen at $-80^{\circ} \mathrm{C}$ for $1 \mathrm{~h}$. (B) GST-RanBP1 fusion proteins on GSH-Sepharose beads were incubated with importin $\beta$ plus Ran. Bound proteins were washed, separated by SDS-PAGE, and immunoblotted for Ran and importin $\beta$ or GST to show that equivalent amounts of the RanBP1 mutants bound to GSH-Sepharose. Molecular weight markers (in kilodaltons) are shown on the left. (C) BHK-21 cells were transfected with wild-type or mutant GFP-RanBP1 plasmids and treated with $200 \mathrm{nM}$ LMB for 30 min, as in Fig. 1, prior to fixation. (D) GFP-BP1(E37K) was transfected into BHK-21 cells. The cells were then either incubated on ice for $1 \mathrm{~h}$ or incubated on ice for 30 min and then treated with $200 \mathrm{nM}$ LMB for an additional 30 min on ice. Fixed cells were stained with DAPI. (E) The C-terminal truncation mutant of Ran BP1(E37K), which lacks the NES, was expressed as a GST fusion protein and injected into the cytosol of BHK-21 cells at a concentration of $1.0 \mathrm{mg} / \mathrm{ml}$ along with $2.0 \mathrm{mg}$ of FITC-dextran/ml as a site-of-injection marker. After $15 \mathrm{~min}$ of incubation at $37^{\circ} \mathrm{C}$, the cells fixed and stained for GST. 


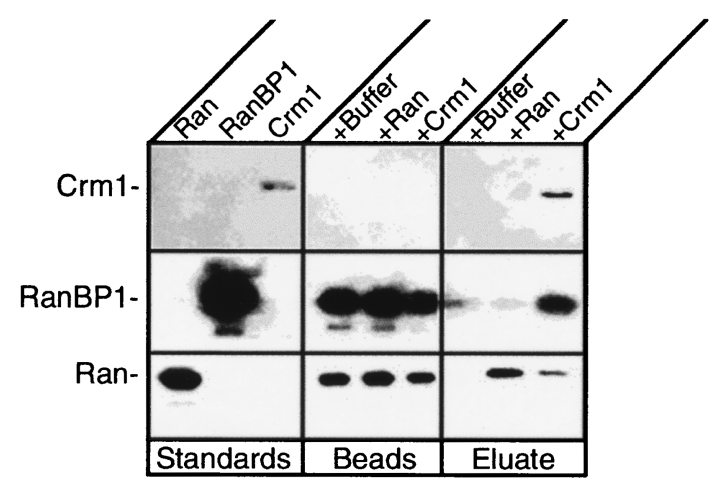

FIG. 10. Crm1 dissociates RanBP1 and Ran from a RanBP1-Ran-importin $\beta$ complex. GST-importin $\beta$ was bound to GSH-Sepharose and incubated with RanBP1 and Ran. Uncomplexed proteins were washed away. The bead-bound complex was then incubated on ice with either buffer, Ran, or Crm1 (29). The eluate and beads were collected separately, subjected to SDS-PAGE, transferred to nitrocellulose, and immunoblotted for Crm1 (top), RanBP1 (middle), and Ran (bottom). Purified proteins were run alongside the beads and eluates as standards.

RanGTP. J. Biol. Chem. 273:33414-33422.

3. Beddow, A. L., S. A. Richards, N. R. Orem, and I. G. Macara. 1995. The Ran/TC4 GTPase-binding domain: identification by expression cloning and characterization of a conserved sequence motif. Proc. Natl. Acad. Sci. USA 92:3328-3332.

4. Bischoff, F. R., and D. Gorlich. 1997. RanBP1 is crucial for the release of RanGTP from importin $\beta$-related nuclear transport factors. FEBS Lett. 419: 249-254.

5. Bischoff, F. R., C. Klebe, J. Kretschmer, A. Wittinghofer, and H. Ponstingl. 1994. RanGAP1 induces GTPase activity of nuclear Ras-related Ran. Proc. Natl. Acad. Sci. USA 91:2587-2591.

6. Bischoff, F. R., H. Krebber, E. Smirnova, W. Dong, and H. Ponstingl. 1995. Co-activation of RanGTPase and inhibition of GTP dissociation by RanGTP binding protein RanBP1. EMBO J. 14:705-715.

7. Bischoff, F. R., and H. Ponstingl. 1991. Catalysis of guanine nucleotide exchange on Ran by the mitotic regulator RCC1. Nature 354:80-82

8. Bischoff, F. R., and H. Ponstingl. 1991. Mitotic regulator protein RCC1 is complexed with a nuclear ras-related polypeptide. Proc. Natl. Acad. Sci. USA 88:10830-10834.

9. Breeuwer, M., and D. S. Goldfarb. 1990. Facilitated nuclear transport of histone H1 and other small nucleophilic proteins. Cell 60:999-1008.

10. Carey, K. L., S. A. Richards, K. M. Lounsbury, and I. G. Macara. 1996. Evidence using a green fluorescent protein-glucocorticoid receptor chimera that the Ran/TC4 GTPase mediates an essential function independent of nuclear protein import. J. Cell Biol. 133:985-996.

11. Chi, N. C., E. J. Adam, and S. A. Adam. 1995. Sequence and characterization of cytoplasmic nuclear protein import factor p97. J. Cell Biol. 130:265-274.

12. Chi, N. C., E. J. H. Adam, and S. A. Adam. 1997. Different binding domains for Ran-Gtp and Ran-Gdp/Ranbp1 on nuclear import factor P97. J. Biol. Chem. 272:6818-6822.

13. Corbett, A. H., D. M. Koepp, G. Schlenstedt, M. S. Lee, A. K. Hopper, and P. A. Silver. 1995. Rna1p, a Ran/TC4 GTPase activating protein, is required for nuclear import. J. Cell Biol. 130:1017-1026.

14. Coutavas, E., M. Ren, J. D. Oppenheim, P. D'Eustachio, and M. G. Rush. 1993. Characterization of proteins that interact with the cell-cycle regulatory protein Ran/TC4. Nature 366:585-587.

15. Davis, L. I. 1995. The nuclear pore complex. Annu. Rev. Biochem. 64:865896.

16. Dingwall, C., and R. A. Laskey. 1991. Nuclear targeting sequences-a consensus? Trends Biochem. Sci. 16:478-481.

17. Drivas, G. T., A. Shih, E. Coutavas, M. G. Rush, and P. D'Eustachio. 1990. Characterization of four novel ras-like genes expressed in a human teratocarcinoma cell line. Mol. Cell. Biol. 10:1793-1798.

18. Englmeier, L., J. C. Olivo, and I. W. Mattaj. 1999. Receptor-mediated substrate translocation through the nuclear pore complex without nucleotide triphosphate hydrolysis. Curr. Biol. 9:30-41.

19. Floer, M., and G. Blobel. 1996. The nuclear transport factor karyopherin beta binds stoichiometrically to Ran-GTP and inhibits the Ran GTPase activating protein. J. Biol. Chem. 271:5313-5316.

20. Floer, M., G. Blobel, and M. Rexach. 1997. Disassembly of RanGTP-karyopherin- $\beta$ complex, an intermediate in nuclear protein import. J. Biol. Chem. 272:19538-19546.

21. Fornerod, M., M. Ohno, M. Yoshida, and I. W. Mattaj. 1997. Crm1 is an export receptor for leucine-rich nuclear export signals. Cell 90:1051-1060.
22. Fukuda, M., S. Asano, T. Nakamura, M. Adachi, M. Yoshida, M. Yanagida, and E. Nishida. 1997. Crm1 is responsible for intracellular transport mediated by the nuclear export signal. Nature 390:308-311.

23. Gerace, L. 1995. Nuclear export signals and the fast track to the cytoplasm. Cell 82:341-344.

24. Gorlich, D. 1998. Transport into and out of the cell nucleus. EMBO J. 17: 2721-2727.

25. Gorlich, D., S. Kostka, R. Kraft, C. Dingwall, R. A. Laskey, E. Hartmann, and S. Prehn. 1995. Two different subunits of importin cooperate to recognize nuclear localization signals and bind them to the nuclear envelope. Curr. Biol. 5:383-392.

26. Gorlich, D., N. Pante, U. Kutay, U. Aebi, and F. R. Bischoff. 1996. Identification of different roles for RanGDP and RanGTP in nuclear protein import. EMBO J. 15:5584-5594.

27. Hellmuth, K., D. M. Lau, F. R. Bischoff, M. Kunzler, E. Hurt, and G. Simos. 1998. Yeast Los1p has properties of an exportin-like nucleocytoplasmic transport factor for tRNA. Mol. Cell. Biol. 18:6374-6386.

28. Hieda, M., T. Tachibana, F. Yokoya, S. Kose, N. Imamoto, and Y. Yoneda. 1999. A monoclonal antibody to the COOH-terminal acidic portion of Ran inhibits both the recycling of Ran and nuclear protein import in living cells. J. Cell Biol. 144:645-655.

29. Holaska, J. M., and B. M. Paschal. 1998. A cytosolic activity distinct from Crm1 mediates nuclear export of protein kinase inhibitor in permeabilized cells. Proc. Natl. Acad. Sci. USA 95:14739-14744.

30. Hopper, A. K., H. M. Traglia, and R. W. Dunst. 1990. The yeast RNA1 gene product necessary for RNA processing is located in the cytosol and apparently excluded from the nucleus. J. Cell Biol. 111:309-321.

31. Imamoto, N., T. Shimamoto, T. Takao, T. Tachibana, S. Kose, M. Matsubae, T. Sekimoto, Y. Shimonishi, and Y. Yoneda. 1995. In vivo evidence for involvement of a $58 \mathrm{kDa}$ component of nuclear pore-targeting complex in nuclear protein import. EMBO J. 14:3617-3626.

32. Izaurralde, E., U. Kutay, C. Vonkobbe, I. W. Mattaj, and D. Gorlich. 1997. The asymmetric distribution of the constituents of the Ran system is essential for transport into and out of the nucleus. EMBO J. 16:6535-6547.

33. Jakel, S., and D. Gorlich. 1998. Importin $\beta$, transportin, RanBP5 and RanBP7 mediate nuclear import of ribosomal proteins in mammalian cells. EMBO J. 17:4491-4502.

34. Kehlenbach, R. H., A. Dickmanns, and L. Gerace. 1998. Nucleocytoplasmic shuttling factors including Ran and Crm1 mediate nuclear export of NFAT in vitro. J. Cell Biol. 141:863-874

35. Kose, S., N. Imamoto, T. Tachibana, T. Shimamoto, and Y. Yoneda. 1997. Ran-unassisted nuclear migration of a 97-kd component of nuclear poretargeting complex. J. Cell Biol. 139:841-849.

36. Kussel, P., and M. Frasch. 1995. Yeast Srp1, a nuclear protein related to Drosophila and mouse pendulin, is required for normal migration, division, and integrity of nuclei during mitosis. Mol. Gen. Genet. 248:351-363.

37. Kutay, U., F. R. Bischoff, S. Kostka, R. Kraft, and D. Gorlich. 1997. Export of importin alpha from the nucleus is mediated by a specific nuclear transport factor. Cell 90:1061-1071.

38. Lounsbury, K. M., and I. G. Macara. 1997. Ran-binding protein 1 (RanBP1) forms a ternary complex with Ran and karyopherin beta and reduces Ran GTPase-activating protein (RanGAP) inhibition by karyopherin beta. J. Biol. Chem. 272:551-555.

39. Lounsbury, K. M., S. A. Richards, K. L. Carey, and I. G. Macara. 1996. Mutations within the Ran/TC4 GTPase-effects on regulatory factor interactions and subcellular localization. J. Biol. Chem. 271:32834-32841.

40. Mattaj, I. W., and L. Englmeier. 1998. Nucleocytoplasmic transport: the soluble phase. Annu. Rev. Biochem. 67:265-306.

41. Mattingly, R. R., A. Sorisky, M. R. Brann, and I. G. Macara. 1994. Muscarinic receptors transform NIH 3T3 cells through a Ras-dependent signalling pathway inhibited by the Ras-GTPase-activating protein SH3 domain. Mol. Cell. Biol. 14:7943-7952.

42. Melchior, F., and L. Gerace. 1998. Two-way trafficking with Ran. Trends Cell Biol. 8:175-179.

43. Nakielny, S., and G. Dreyfuss. 1998. Import and export of the nuclear protein import receptor transportin by a mechanism independent of GTP hydrolysis. Curr. Biol. 8:89-95.

44. Ossarehnazari, B., F. Bachelerie, and C. Dargemont. 1997. Evidence for a role of Crm1 in signal-mediated nuclear protein export. Science 278:141144.

45. Paschal, B. M., C. Fritze, T. Guan, and L. Gerace. 1997. High levels of the GTPase Ran/TC4 relieve the requirement for nuclear protein transport factor 2. J. Biol. Chem. 272:21534-21539.

46. Pasquinelli, A. E., M. A. Powers, E. Lund, D. Forbes, and J. E. Dahlberg. 1997. Inhibition of mRNA export in vertebrate cells by nuclear export signal conjugates. Proc. Natl. Acad. Sci. USA 94:14394-14399.

47. Petersen, C., N. Orem, J. Trueheart, J. Thorner, and I. G. Macara. 2000. Random mutagenesis and functional analysis of the Ran binding protein, RanBP1. J. Biol. Chem. 275:4081-4091.

48. Radu, A., G. Blobel, and M. S. Moore. 1995. Identification of a protein complex that is required for nuclear protein import and mediates docking of 
import substrate to distinct nucleoporins. Proc. Natl. Acad. Sci. USA 92: 1769-1773.

49. Rexach, M., and G. Blobel. 1995. Protein import into nuclei: association and dissociation reactions involving transport substrate, transport factors, and nucleoporins. Cell 83:683-692.

50. Ribbeck, K., U. Kutay, E. Paraskeva, and D. Gorlich. 1999. The translocation of transportin-cargo complexes through nuclear pores is independent of both ran and energy. Curr. Biol. 9:47-50.

51. Ribbeck, K., G. Lipowsky, H. M. Kent, M. Stewart, and D. Gorlich. 1998. NTF2 mediates nuclear import of Ran. EMBO J. 17:6587-6598.

52. Richards, S. A., K. L. Carey, and I. G. Macara. 1997. Requirement of guanosine triphosphate-bound Ran for signal-mediated nuclear protein export. Science 276:1842-1844.

53. Richards, S. A., K. M. Lounsbury, K. L. Carey, and I. G. Macara. 1996. A nuclear export signal is essential for the cytosolic localization of the Ran binding protein, RanBP1. J. Cell Biol. 134:1157-1168.

54. Richards, S. A., K. M. Lounsbury, and I. G. Macara. 1995. The C terminus of the nuclear Ran/TC4 GTPase stabilizes the GDP-bound state and mediates interactions with RCC1, Ran-GAP, and HTF9A/RanBP1. J. Biol. Chem. 270:14405-14411.

55. Scheffzek, K., C. Klebe, K. Fritz-Wolf, W. Kabsch, and A. Wittinghofer. 1995. Crystal structure of the nuclear Ras-related protein Ran in its GDP-bound form. Nature 374:378-381.

56. Schlenstedt, G., E. Smirnova, R. Deane, J. Solsbacher, U. Kutay, D. Gorlich, H. Ponstingl, and F. R. Bischoff. 1997. Yrb4p, a yeast Ran-GTP-binding protein involved in import of ribosomal protein L25 into the nucleus. EMBO J. 16:6237-6249.

57. Schlenstedt, G., D. H. Wong, D. M. Koepp, and P. A. Silver. 1995. Mutants in a yeast Ran binding protein are defective in nuclear transport. EMBO J. 14:5367-5378.

58. Seki, T., N. Hayashi, and T. Nishimoto. 1996. RCC1 in the Ran pathway. J. Biochem. 120:207-214
59. Smith, A., A. Brownawell, and I. G. Macara. 1998. Nuclear import of Ran: GDP is mediated by NTF2. Curr. Biol. 8:1403-1406.

60. Stade, K., C. S. Ford, C. Guthrie, and K. Weis. 1997. Exportin 1 (Crm1p) is an essential nuclear export factor. Cell 90:1041-1050.

61. Torok, I., D. Strand, R. Schmitt, G. Tick, T. Torok, I. Kiss, and B. M. Mechler. 1995. The overgrown hematopoietic organs-31 tumor suppressor gene of Drosophila encodes an importin-like protein accumulating in the nucleus at the onset of mitosis. J. Cell Biol. 129:1473-1489.

62. Vetter, I. R., C. Nowak, T. Nishimoto, J. Kuhlmann, and A. Wittinghofer. 1999. Structure of a Ran-binding domain complexed with Ran bound to a GTP analogue: implications for nuclear transport. Nature 398:39-46.

63. Wolff, B., J. J. Sanglier, and Y. Wang. 1997. Leptomycin B is an inhibitor of nuclear export-inhibition of nucleo-cytoplasmic translocation of the human immunodeficiency virus type 1 (HIV-1) Rev protein and Rev-dependent mRNA. Chem. Biol. 4:139-147.

64. Wozniak, R. W., M. P. Rout, and J. D. Aitchison. 1998. Karyopherins and kissing cousins. Trends Cell Biol. 8:184-188.

65. Wu, J., M. J. Matunis, D. Kraemer, G. Blobel, and E. Coutavas. 1995. Nup358, a cytoplasmically exposed nucleoporin with peptide repeats, RanGTP binding sites, zinc fingers, a cyclophilin A homologous domain, and a leucine-rich region. J. Biol. Chem. 270:14209-14213.

66. Yang, Q., M. P. Rout, and C. W. Akey. 1998. Three-dimensional architecture of the isolated yeast nuclear pore complex-functional and evolutionary implications. Mol. Cell 1:223-234.

67. Yokoyama, N., N. Hayashi, T. Seki, N. Pante, T. Ohba, K. Nishii, K. Kuma, T. Hayashida, T. Miyata, U. Aebi, et al. 1995. A giant nucleopore protein that binds Ran/TC4. Nature 376:184-188.

68. Zolotukhin, A. S., and B. K. Felber. 1997. Mutations in the nuclear export signal of human Ran-binding protein RanBP1 block the Rev-mediated posttranscriptional regulation of human immunodeficiency virus type 1 . J. Biol. Chem. 272:11356-11360. 